\title{
Structure Preserving Bisimilarity, Supporting an Operational Petri Net Semantics of CCSP
}

\author{
Rob J. van Glabbeek ${ }^{12}$ \\ 1 NICTA $^{\star}$, Sydney, Australia \\ ${ }^{2}$ Computer Science and Engineering, UNSW, Sydney, Australia
}

\begin{abstract}
In 1987 Ernst-Rüdiger Olderog provided an operational Petri net semantics for a subset of CCSP, the union of Milner's CCS and Hoare's CSP. It assigns to each process term in the subset a labelled, safe place/transition net. To demonstrate the correctness of the approach, Olderog established agreement (1) with the standard interleaving semantics of CCSP up to strong bisimulation equivalence, and (2) with standard denotational interpretations of CCSP operators in terms of Petri nets up to a suitable semantic equivalence that fully respects the causal structure of nets. For the latter he employed a linear-time semantic equivalence, namely having the same causal nets.

This paper strengthens (2), employing a novel branching-time version of this semantics-structure preserving bisimilarity - that moreover preserves inevitability. I establish that it is a congruence for the operators of CCSP.
\end{abstract}

\section{Introduction}

The system description languages CCS and CSP have converged to one theory of processes which—following a suggestion of M. Nielsen—was called "CCSP" in [26]. The standard semantics of this language is in terms of labelled transition systems modulo strong bisimilarity, or some coarser semantic equivalence. In the case of CCS, a labelled transition system is obtained by taking as states the closed CCS expressions, and as transitions those that are derivable from a collection of rules by induction on the structure of these expressions [24]; this is called a (structural) operational semantics [30]. The semantics of CSP was originally given in quite a different way [3|20], but [28] provided an operational semantics of CSP in the same style as the one of CCS, and showed its consistency with the original semantics.

Such semantics abstract from concurrency relations between actions by reducing concurrency to interleaving. An alternative semantics, explicitly modelling concurrency relations, requires models like Petri nets [33] or event structures [25[36]. In [36|21] non-interleaving semantics for variants of CCSP are given in terms of event structures. However, infinite event structures are needed to model simple systems involving loops, whereas Petri nets, like labelled transition systems, offer finite representations for some such systems. Denotational semantics in terms of Petri nets of the essential CCSP operators are given in [18]35|16] - see [27] for more references. Yet a satisfactory denotational Petri net semantics treating recursion has to my knowledge not been proposed.

\footnotetext{
* NICTA is funded by the Australian Government through the Department of Communications and the Australian Research Council through the ICT Centre of Excellence Program.
} 
Olderog [26]27] closed this gap by giving an operational net semantics in the style of [30|24] for a subset of CCSP including recursion-to be precise: guarded recursion. To demonstrate the correctness of his approach, Olderog proposed two fundamental properties such a semantics should have, and established that both of them hold [27]:

- Retrievability. The standard interleaving semantics for process terms should be retrievable from the net semantics.

- Concurrency. The net semantics should represent the intended concurrency of process terms.

The second requirement was not met by an earlier operational net semantics from [5].

To formalise the first requirement, Olderog notes that a Petri net induces a labelled transition system through the firing relation between markings- the interleaving case graph - and requires that the interpretation of any CCSP expression as a state in a labelled transition system through the standard interleaving semantics of CCSP should be strongly bisimilar to the interpretation of this expression as a marking in the interleaving case graph induced by its net semantics.

To formalise the second requirement, he notes that the intended concurrency of process terms is clearly represented in the standard denotational semantics of CCSP operators [18 35 16], and thus requires that the result of applying a CCSP operator to its arguments according to this denotational semantics yields a similar result as doing this according to the new operational semantics. The correct representation of recursion follows from the correct representation of the other operators through the observation that a recursive call has the very same interpretation as a Petri net as its unfolding.

A crucial parameter in this formalisation is the meaning of "similar". A logical choice would be semantic equivalence according to one of the non-interleaving equivalences found in the literature, where a finer or more discriminating semantics gives a stronger result. To match the concurrency requirement, this equivalence should respect concurrency, in that it only identifies nets which display the same concurrency relations. In this philosophy, the semantics of a CCSP expression is not so much a Petri net, but a semantic equivalence class of Petri nets, i.e. a Petri net after abstraction from irrelevant differences between nets. For this idea to be entirely consistent, one needs to require that the chosen equivalence is a congruence for all CCSP constructs, so that the meaning of the composition of two systems, both represented as equivalence classes of nets, is independent of the choice of representative Petri nets within these classes.

Instead of selecting such an equivalence, Olderog instantiates "similar" in the above formalisation of the second requirement with strongly bisimilar, a new relation between nets that should not be confused with the traditional relation of strong bisimilarity between labelled transition systems. As shown in [1], strong bisimilarity fails to be an equivalence: it is reflexive and symmetric, but not transitive.

As pointed out in [27, Page 37] this general shortcoming of strong bisimilarity "does not affect the purpose of this relation" in that book: there it "serves as an auxiliary notion in proving that structurally different nets are causally equivalent". Here causal equivalence means having the same causal nets, where causal nets [29]34] model concurrent computations or executions of Petri nets. So in effect Olderog does choose a semantic equivalence on Petri nets, namely having the same concurrent computations as modelled by causal nets. This equivalence fully respects concurrency. 


\subsection{Structure preserving bisimilarity}

The contribution of the present paper is a strengthening of this choice of a semantic equivalence on Petri nets. I propose the novel structure preserving bisimulation equivalence on Petri nets, and establish that the result of applying a CCSP operator to its arguments according to the standard denotational semantics yields a structure preserving bisimilar result as doing this according to Olderog's operational semantics. The latter is an immediate consequence of the observation that structure preserving bisimilarity between two nets is implied by Olderog's strong bisimilarity.

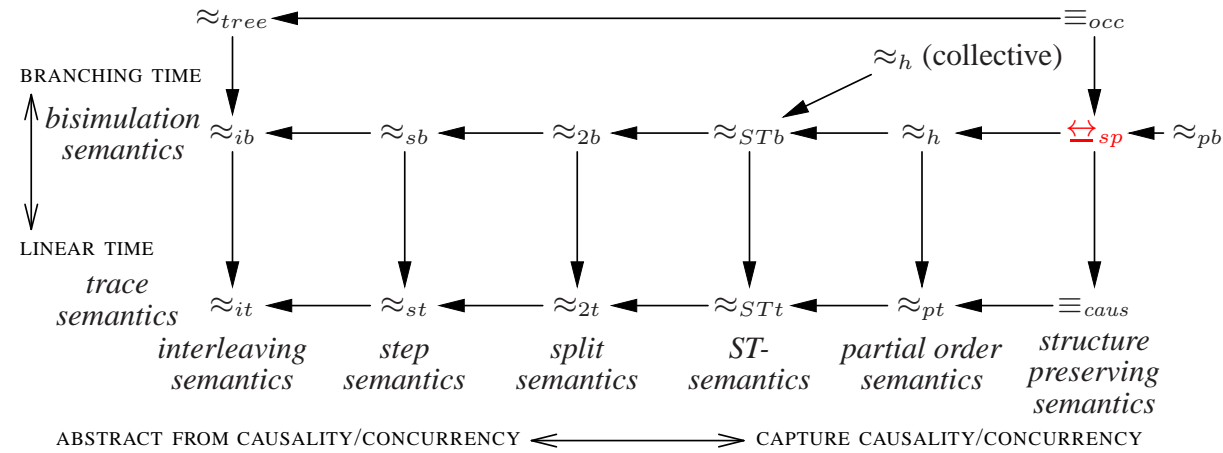

Fig. 1. A spectrum of semantic equivalences on Petri nets

Figure 1 shows a map of some equivalence relations on nets found in the literature, in relation to the new structure preserving bisimilarity, $\overleftrightarrow{s}_{s p}$. The equivalences become finer when moving up or to the right; thus coarser or less discriminating when following the arrows. The rectangle from $\approx_{i t}$ to $\approx_{h}$ is taken from [10]. The vertical axis is the linear time - branching time spectrum, with trace equivalence at the bottom and (strong) bisimulation equivalence, or bisimilarity, at the top. A host of intermediate equivalences is discussed in [11]. The key difference is that linear time equivalences, like trace equivalence, only consider the set of possible executions of a process, whereas branching time equivalences, like bisimilarity, additionally take into account at which point the choice between two executions is made. The horizontal axis indicates to what extent concurrency information is taken into account. Interleaving equivalences-on the left—fully abstract from concurrency by reducing it to arbitrary interleaving; step equivalences additionally take into account the possibility that two concurrent actions happen at exactly the same moment; split equivalences recognise the beginning and end of actions, which here are regarded to be durational, thereby capturing some information about their overlap in time; $S T$ - or interval equivalences fully capture concurrency information as far as possible by considering durational actions overlapping in time; and partial order equivalences capture the causal links between actions, and thereby all concurrency. By taking the product of these two axes, one obtains a two-dimensional spectrum of equivalence relations, with entries like interleaving bisimulation equivalence $\approx_{i b}$ and partial order trace equivalence $\approx_{p t}$. For the right upper corner several partial order bisimulation equivalences were proposed in the literature; according to [13] 
the history preserving bisimulation equivalence $\approx_{h}$, originally proposed by [32], is the coarsest one that fully captures the interplay between causality and branching time.

The causal equivalence employed by Olderog, $\equiv_{\text {caus }}$, is a linear time equivalence strictly finer than $\approx_{p t}$. Since it preserves information about the number of preplaces of a transition, it is specific to a model of concurrency based on Petri nets; i.e. there is no obvious counterpart in terms of event structures. I found only two equivalences in the literature that are finer than both $\equiv_{\text {caus }}$ and $\approx_{h}$, namely occurrence net equivalence [16]— $\equiv_{o c c}$-and the place bisimilarity $\approx_{p b}$ of [1]. Two nets are occurrence net equivalent iff they have isomorphic unfoldings. The unfolding, defined in [25], associates with a given safe Petri net $N$ a loop-free net—an occurrence net-that combines all causal nets of $N$, together with their branching structure. This unfolding is similar to the unfolding of a labelled transition system into a tree, and thus the interleaving counterpart of occurrence net equivalence is tree equivalence [11], identifying two transition systems iff their unfoldings are isomorphic. The place bisimilarity was inspired by Olderog's strong bisimilarity, but adapted to make it transitive, and thus an equiva-

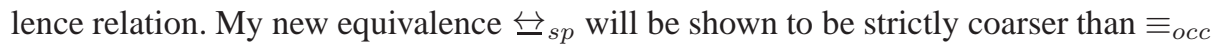
and $\approx_{p b}$, yet finer than both $\equiv_{\text {caus }}$ and $\approx_{h}$.

The equivalences discussed above (without the diagonal line in Figure 1) are all defined on safe Petri nets. Additionally, the definitions generalise to unsafe Petri nets. However, there are two possible interpretations of unsafe Petri nets, called the collective token and the individual token interpretation [12], and this leads to two versions of history preserving bisimilarity. The history preserving bisimilarity based on the individual token interpretation was first defined for Petri nets in [2], under the name fully concurrent bisimulation equivalence. At the level of ST-semantics the collective and individual token interpretations collapse. The unfolding of unsafe Petri nets, and thereby occurrence net equivalence, has been defined for the individual token interpretation only [723]12], and likewise causal equivalence can be easily generalised within the individual token interpretation. The new structure preserving bisimilarity falls in the individual token camp as well.

\subsection{Criteria for choosing this semantic equivalence}

In selecting a new semantic equivalence for reestablishing Olderog's agreement of operational and denotational interpretations of CCSP operators, I consider the following requirements on such a semantic equivalence (with subsequent justifications):

1. it should be a branching time equivalence,

2. it should fully capture causality relations and concurrency (and the interplay between causality and branching time),

3. it should respect inevitability [22], meaning that if two systems are equivalent, and in one the occurrence of a certain action is inevitable, then so is it in the other,

4. it should be real-time consistent [16], meaning that for every association of execution times to actions, assuming that actions happen as soon as they can, the running times associated with computations in equivalent systems should be the same,

5. it should be preserved under action refinement [4]13], meaning that if in two equivalent Petri nets the same substitutions of nets for actions are made, the resulting nets should again be equivalent, 
6. it should be finer than Olderog's causal equivalence,

7. it should not distinguish systems whose behaviours are patently the same, such as Petri nets that differ only in their unreachable parts,

8. it should be a congruence for the constructs of CCSP,

9. and it should allow to establish agreement between the operational and denotational interpretations of CCSP operators.

Requirement 1 is the driving force behind this contribution. It is motivated by the insight that branching time equivalences better capture phenomena like deadlock behaviour. Since in general a stronger result on the agreement between operational and denotational semantics is obtained when employing a finer semantics, I aim for a semantics that fully captures branching time information, and thus is at least as discriminating as interleaving bisimilarity.

Requirement 2 is an obvious choice when the goal of the project is to capture concurrency explicitly. The combination of Requirements 1 and 2 then naturally asks for an equivalence that is at least as fine as $\approx_{h}$. One might wonder, however, for what reason one bothers to define a semantics that captures concurrency information. In the literature, various practical reasons have been given for preferring a semantics that (partly) respects concurrency and causality over an interleaving semantics. Three of the more prominent of these reasons are formulated as requirements 3, 4 and 5 above.

Requirement 3 is manifestly useful when considering liveness properties of systems. Requirement 4 obviously has some merit when timing is an issue. Requirement 5 is useful in system design based on stepwise refinement [13].

Requirement 6 is only there so that I can truthfully state to have strengthened Olderog's agreement between the denotational and operational semantics, which was stated in terms of causal equivalence. This requirement will not be needed in my justification for introducing a new semantic equivalence-and neither will Requirement 2 .

Requirement 7 is hardly in need of justification. The paper [1] lists as a desirable property of semantic equivalences - one that is not met by their own proposal $\approx_{p b}$ that they should not distinguish nets that have isomorphic unfoldings, given that unfolding a net should not be regarded as changing it behaviour. When working within the individual token interpretation of nets I will take this as a suitable formalisation of Requirement 7.

The argument for Requirement 8 has been given earlier in this introduction, and Requirement 9 underlies my main motivation for selecting a semantic equivalence in the first place.

\subsection{Applying the criteria}

Table 1 tells which of these requirements are satisfied by the semantic equivalences from Section 1.1 (not considering the one collective token equivalence there). The first two rows, reporting which equivalences satisfy Requirements 1 and 2, are well-known; these results follow directly from the definitions. The third row, reporting on respect for inevitability, is a contribution of this paper, and will be discussed in Section 1.4 and delivered in Sections 11,14 
Table 1. Which requirements are satisfied by the various semantic equivalences

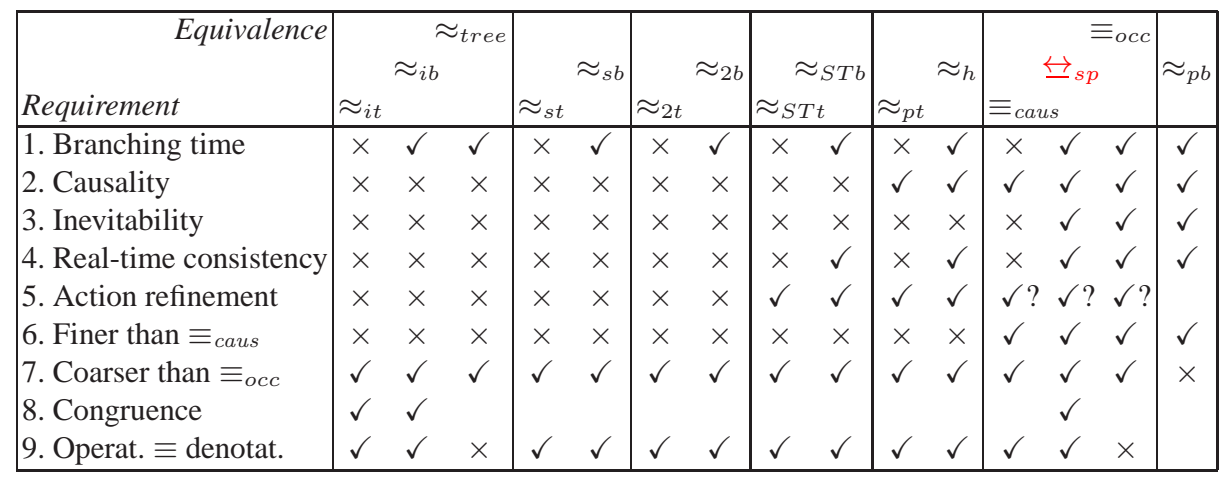

Regarding Row 4, In [16] it is established that ST-bisimilarity is real-time consistent. Moreover, the formal definition is such that if a semantic equivalence $\approx$ is realtime consistent, then so is any equivalence finer than $\approx$. Linear time equivalences are not real-time consistent, and neither is $\approx_{2 b}$ [17].

In [13] it is established that $\approx_{p t}$ and $\approx_{h}$ are preserved under action refinement, but interleaving and step equivalences are not, because they do not capture enough information about concurrency. In [10] it is shown that $\approx_{S T t}$ and $\approx_{S T b}$ are already preserved under action refinement, whereas by [17] split semantics are not. I conjecture that $\equiv_{c a u s}$ and $\equiv_{o c c}$ are also preserved under action refinement, but I have not seen a

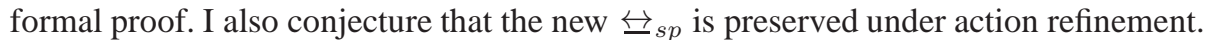

Rows 6 and 7 follow as soon as I have formally established the implications of

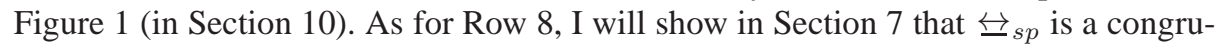
ence for the operators of CCSP. That also $\approx_{i t}$ and $\approx_{i b}$ are congruences for CCSP is well known. The positive results in Row 9 follow from the fact that Olderog's strong

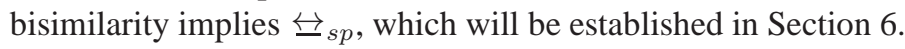

Requirements 1 and 6 together limit the search space for suitable equivalence relations to $\equiv_{o c c}, \approx_{p b}$ and the new $\overleftrightarrow{ }_{s p}$. When dropping Requirement 6 , but keeping 2 , also $\approx_{h}$ becomes in scope. When also dropping 2 , but keeping 4 , I gain $\approx_{S T b}$ as a candidate equivalence. However, both $\approx_{h}$ and $\approx_{S T b}$ will fall pray to Requirement 3 , so also without Requirements 2 and 6 the search space will be limited to $\equiv_{o c c}, \approx_{p b}$ and the new $\overleftrightarrow{ }_{s p}$.

Requirement 7 rules out $\approx_{p b}$, as that equivalence makes distinctions based on unreachable parts of nets [1]. The indispensable Requirement 9 rules out $\equiv_{o c c}$, since that equivalence distinguishes the operational and denotational semantics of the CCSP expression $a 0+a 0$. According to the operational semantics this expression has only one transition, whereas by the denotational semantics it has two, and $\equiv_{o c c}$ does not collapse identical choices. The same issue plays in interleaving semantics, where the operational and denotational transition system semantics of CCSP do not agree up to tree equivalence. This is one of the main reasons that bisimilarity is often regarded as the top of the linear time - branching time spectrum.

This constitutes the justification for the new equivalence $\overleftrightarrow{ }_{s p}$. 


\subsection{Inevitability}

The meaning of Requirement 3 depends on which type of progress or fairness property one assumes to guarantee that actions that are due to occur will actually happen. Lots of fairness assumption are mentioned in the literature, but, as far as I can tell, they can be classified in exactly 4 groups: progress, justness, weak fairness and strong fairness [15]. These four groups form a hierarchy, in the sense that one cannot consistently assume strong fairness while objecting to weak fairness, or justness while objecting to progress.

Strong and weak fairness deal with choices that are offered infinitely often. Suppose you have a shop with only two customers $A$ and $B$ that may return to the shop to buy something else right after they are served. Then it is unfair to only serve customer $A$ again and again, while $B$ is continuously waiting to be served. In case $B$ is not continuously ready to be served, but sometimes goes home to sleep, yet always returns to wait for his turn, it is weakly fair to always ignore customer $B$ in favour of $A$, but not strongly fair.

Weak and strong fairness assumptions can be made locally, pertaining to some repeating choices of the modelled system but not to others, or globally, pertaining to all choices of a given type. Since the real world is largely unfair, strong and weak fairness assumptions need to be made with great caution, and they will not appear in this paper.

Justness and progress assumptions, on the other hand, come only in the global variant, and can be safely assumed much more often. A progress assumption says that if a system can do some action (that is not contingent on external input) it will do an action. In the example of the shop, if there is a customer continuously ready to be served, and the clerk stands pathetically behind the counter staring at the customer but not serving anyone, there is a failure of progress. Without assuming progress, no action is inevitable, because it is always possible that a system will remain in its initial state without ever doing anything. Hence the concept of inevitability only makes sense when assuming at least progress.

Justness [8]15] says roughly that if a parallel component can make progress (not contingent on input from outside of this component) it will do so. Suppose the shop has two counters, each manned by a clerk, and, whereas customer $A$ is repeatedly served at counter 1 , customer $B$ is ready to be served by counter 2 , but is only stared at by a pathetic clerk. This is not a failure of progress, as in any state of the system someone will be served eventually. Yet it counts as a failure of justness. In the context of Petri nets, a failure of justness can easily be formalised as an execution, during which, from some point onwards, all preplaces of a given transition remain marked, yet the transition never fires [14]. One could argue that, when taking concurrency seriously, justness should be assumed whenever one assumes progress.

Inevitability can be easily expressed in temporal logics like LTL [31] or CTL [6], and it is well known that strongly bisimilar transition systems satisfy the same temporal formulas. This suggests that interleaving bisimilarity already respects inevitability. However, this conclusion is warranted only when assuming progress but not justness, or perhaps also when assuming some form of weak or strong fairness. The system $C:=\langle X \mid X=a X+b X\rangle$ - using the CCSP syntax of Section 2 - repeatedly choosing between the actions $a$ and $b$, is interleaving bisimilar to the system $D:=$ $\langle Y \mid Y=a Y\rangle \|\langle Z \mid Z=b Z\rangle$, which in parallel performs infinitely many $a$ s and infinitely 
many $b s$. Yet, when assuming justness but not weak fairness, the execution of the action $b$ is inevitable in $D$, but not in $C$. This shows that when assuming justness but not weak fairness, interleaving bisimilarity does not respect inevitability. The paper [22], which doesn't use Petri nets as system model, leaves the precise formulation of a justness assumption for future work-this task is undertaken in the different context of CCS in [15]. Also, respect of inevitability as a criterion for judging semantic equivalences does not occur in [22], even though "the partial order approach" is shown to be beneficial.

In this paper, assuming justness but not strong or weak fairness, I show that neither $\approx_{h}$ nor $\equiv_{\text {caus }}$ respects inevitability (using infinite nets in my counterexample). Hence, respecting concurrency appears not quite enough to respect inevitability. Respect for inevitability, like real-time consistency, is a property that holds for any equivalence relation finer than one for which it is known to hold already. So also none of the ST- or

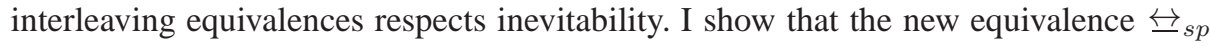
respects inevitability. This makes it the coarsest equivalence of Figure 1 that does so.

\section{CCSP}

CCSP is parametrised by the choice of an infinite set Act of actions, that I will assume to be fixed for this paper. Just like the version of CSP from Hoare [20], the version of CCSP used here is a typed language, in the sense that with every CCSP process $P$ an explicit alphabet $\alpha(P) \subseteq A c t$ is associated, which is a superset of the set of all actions the process could possibly perform. This alphabet is exploited in the definition of the parallel composition $P \| Q$ : actions in the intersection of the alphabets of $P$ and $Q$ are required to synchronise, whereas all other actions of $P$ and $Q$ happen independently. Because of this, processes with different alphabets may never be identified, even if they can perform the same set of actions and are alike in all other aspects. It is for this reason that I interpret CCSP in terms of typed Petri nets, with an alphabet as extra component.

I also assume an infinite set $V$ of variable names. A variable is a pair $X_{A}$ with $X \in V$ and $A \subseteq$ Act. The syntax of (my subset of) CCSP is given by

$$
P::=0_{A}|a P| P+P|P \| P| R(P)\left|X_{A}\right|\left\langle X_{A} \mid \mathcal{S}\right\rangle\left(\text { with } X_{A} \in V_{\mathcal{S}}\right)
$$

with $A \subseteq A c t, a \in A c t, R \subseteq A c t \times A c t, X \in V$ and $\mathcal{S}$ a recursive specification: a set of equations $\left\{Y_{B}=\mathcal{S}_{Y_{B}} \mid Y_{B} \in V_{\mathcal{S}}\right\}$ with $V_{\mathcal{S}} \subseteq V \times$ Act (the bound variables of $\mathcal{S}$ ) and $\mathcal{S}_{Y_{B}}$ a CCSP expression satisfying $\alpha\left(\mathcal{S}_{Y_{B}}\right)=B$ for all $Y_{B} \in V_{\mathcal{S}}$ (were $\alpha\left(\mathcal{S}_{Y_{B}}\right)$ is defined below). The constant $0_{A}$ represents a process that is unable to perform any action. The process $a P$ first performs the action $a$ and then proceeds as $P$. The process $P+Q$ will behave as either $P$ or $Q, \|$ is a partially synchronous parallel composition operator, $R$ a renaming, and $\left\langle X_{A} \mid \mathcal{S}\right\rangle$ represents the $X_{A^{-}}$-component of a solution of the system of recursive equations $\mathcal{S}$. A CCSP expression $P$ is closed if every occurrence of a variable $X_{A}$ occurs in a subexpression $\left\langle Y_{B} \mid \mathcal{S}\right\rangle$ of $P$ with $X_{A} \in V_{\mathcal{S}}$.

The constant 0 and the variables are indexed with an alphabet. The alphabet of an arbitrary CCSP expression is given by:

- $\alpha\left(0_{A}\right)=\alpha\left(X_{A}\right)=\alpha\left(\left\langle X_{A} \mid \mathcal{S}\right\rangle\right)=A$

- $\alpha(a P)=\{a\} \cup \alpha(P)$ 
Table 2. Structural operational interleaving semantics of CCSP

\begin{tabular}{|ccc|}
\hline$a P \stackrel{a}{\rightarrow} P$ & $\frac{P \stackrel{a}{\rightarrow} P^{\prime}}{P\left\|Q \stackrel{a}{\longrightarrow} P^{\prime}\right\| Q}(a \notin \alpha(Q))$ & $\frac{P \stackrel{a}{\longrightarrow} P^{\prime}}{R(P) \stackrel{b}{\longrightarrow} R\left(P^{\prime}\right)}((a, b) \in R)$ \\
$\frac{P \stackrel{a}{\rightarrow} P^{\prime}}{P+Q \stackrel{a}{\longrightarrow} P^{\prime}}$ & $\frac{P \stackrel{a}{\longrightarrow} P^{\prime}, Q \stackrel{a}{\longrightarrow} Q^{\prime}}{P\left\|Q \stackrel{a}{\longrightarrow} P^{\prime}\right\| Q^{\prime}}(a \in \alpha(P) \cap \alpha(Q))$ \\
$\frac{a}{\longrightarrow} Q^{\prime}$ & $\frac{Q \stackrel{a}{\longrightarrow} Q^{\prime}}{P\|Q \stackrel{a}{\longrightarrow} P\| Q^{\prime}}(a \notin \alpha(P))$ & $\frac{\left\langle\mathcal{S}_{X_{A}} \mid \mathcal{S}\right\rangle \stackrel{a}{\longrightarrow} P^{\prime}}{\left\langle X_{A} \mid \mathcal{S}\right\rangle \stackrel{a}{\rightarrow} P^{\prime}}$ \\
\hline
\end{tabular}

- $\alpha(P+Q)=\alpha(P \| Q)=\alpha(P) \cup \alpha(Q)$

- $\alpha(R(P))=\{b \mid \exists a \in \alpha(P):(a, b) \in R\}$.

Substitutions of expressions for variables are allowed only if the alphabets match. For this reason a recursive specification $\mathcal{S}$ is declared syntactically incorrect if $\alpha\left(\mathcal{S}_{Y_{B}}\right) \neq B$ for some $Y_{B} \in V_{\mathcal{S}}$. The interleaving semantics of CCSP is given by the labelled transition relation $\rightarrow \subseteq \mathrm{T}_{\mathrm{CCSP}} \times$ Act $\times \mathrm{T}_{\mathrm{CCSP}}$ on the set $\mathrm{T}_{\mathrm{CCSP}}$ of closed CCSP terms, where the transitions $P \stackrel{a}{\longrightarrow} Q$ (on arbitrary CCSP expressions) are derived from the rules of Table 2. Here $\langle P \mid \mathcal{S}\rangle$ for $P$ an expression and $\mathcal{S}$ a recursive specification denotes the expression $P$ in which $\left\langle Y_{B} \mid \mathcal{S}_{Y_{B}}\right\rangle$ has been substituted for the variable $Y_{B}$ for all $Y_{B} \in V_{\mathcal{S}}$.

A CCSP expression is well-typed if for any subexpression of the form $a P$ one has $a \in \alpha(P)$ and for any subexpression of the form $P+Q$ one has $\alpha(P)=\alpha(Q)$. Thus $a 0_{\{a\}}+b X_{\emptyset}$ is not well-typed, although the equivalent expression $a 0_{\{a, b\}}+b X_{\{a, b\}}$ is. A recursive specification $\left\langle X_{A} \mid \mathcal{S}\right\rangle$ is guarded if each occurrence of a variable $Y_{B} \in V_{\mathcal{S}}$ in a term $\mathcal{S}_{Z_{C}}$ for some $Z_{C} \in V_{\mathcal{S}}$ lays within a subterm of $\mathcal{S}_{Z_{C}}$ of the form $a P$. Following [27] I henceforth only consider well-typed CCSP expressions with guarded recursion.

In Olderog's subset of CCSP, each recursive specification has only one equation, and renamings must be functions instead of relations. Here I allow mutual recursion and relational renaming, where an action may be renamed into a choice of several actionsor possibly none. This generalisation does not affect any of the proofs in [27].

Example 1. The behaviour of the customer from Section 1.4 could be given by the recursive specification $\mathcal{S}_{\text {CUs }}$ :

$$
\text { CUS }_{C u}=\text { enter buy leave } \operatorname{CUS}_{C u}
$$

indicating that the customer keeps coming back to the shop to buy more things. Here enter, buy, leave $\in$ Act and CUs $\in V$. The customer's alphabet $C u$ is $\{$ enter, buy, leave $\}$. Likewise, the behaviour of the store clerk could be given by the specification $\mathcal{S}_{\text {CLK }}$ :

$$
\mathrm{CLK}_{C l}=\text { serve } \mathrm{CLK}_{C l}
$$

where $C l=\{$ serve $\}$. The CCSP processes representing the customer and the clerk, with their reachable states and labelled transitions between them, are displayed in Figure 2 

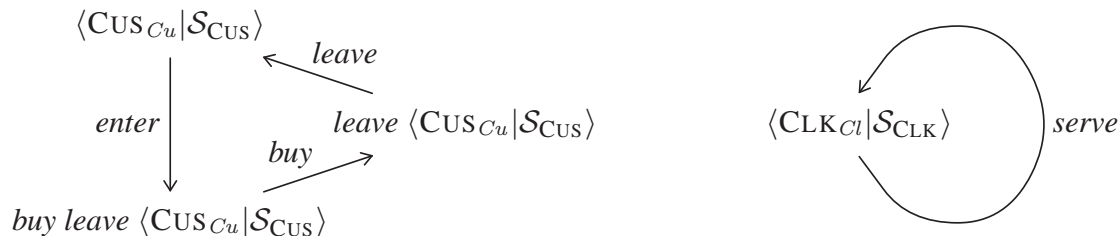

Fig. 2. Labelled transition semantics of customer and clerk

In order to ensure that the parallel composition synchronises the buy-action of the customer with the serve-action of the clerk, I apply renaming operators $R_{\mathrm{CUS}}$ and $R_{\mathrm{CLK}}$ with $R_{\mathrm{Cus}}($ buy $)=$ serves and $R_{\mathrm{CLK}}($ serve $)=$ serves and leaving all other actions unchanged, where serves is a joint action of the renamed customer and the renamed clerk. The total CCSP specification of a store with one clerk and one customer is

$$
R_{\mathrm{Cus}}\left(\left\langle\mathrm{CUS}_{C u} \mid \mathcal{S}_{\mathrm{CUS}}\right\rangle\right) \| R_{\mathrm{CLK}}\left(\left\langle\mathrm{CLK}_{C l} \mid \mathcal{S}_{\mathrm{CLK}}\right\rangle\right)
$$

and the relevant part of the labelled transition system of CCSP is displayed below.

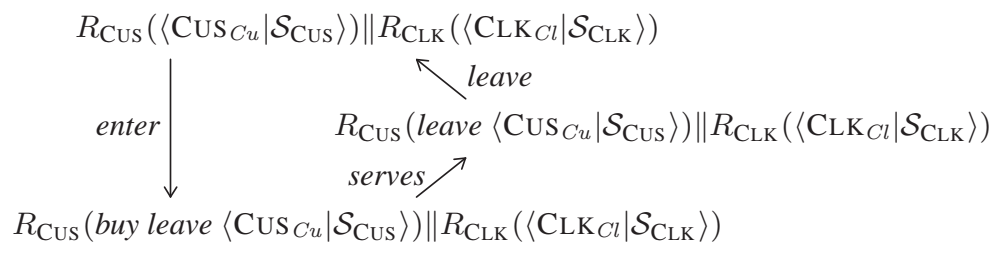

Fig. 3. Labelled transition semantics of the 1-customer 1-clerk store

One possible behaviour of this system is the sequence of actions enter serves leave enter, followed by eternal stagnation. This behaviour is ruled out by the progress assumption of Section 1.4. The only behaviour compatible with this assumption is the infinite sequence of actions (enter serves leave $)^{\infty}$.

To model a store with two customers (A and B) and 2 clerks (I and II), I introduce a relational renaming for each of them, defined by

$$
\begin{array}{ll}
R_{A}(\text { enter })=\text { A enters } & R_{A}(\text { buy })=\{\mathrm{I} \text { serves } A, \mathrm{II} \text { serves } A\} \quad R_{A}(\text { leave })=\text { Aleaves } \\
R_{B}(\text { enter })=B \text { enters } & R_{B}(\text { buy })=\{\mathrm{I} \text { serves } B, \mathrm{II} \text { serves } B\} \quad R_{B}(\text { leave })=B \text { leaves } \\
& R_{\mathrm{I}}(\text { serve })=\{\mathrm{I} \text { serves } A, \mathrm{I} \text { serves } B\} \\
& R_{\mathrm{II}}(\text { serve })=\{\mathrm{II} \text { serves } A, \mathrm{II} \text { serves } B\} .
\end{array}
$$

The CCSP specification of a store with two clerks and two customers is

$$
\left(R_{A}\left(\left\langle\operatorname{Cus}_{C u} \mid \mathcal{S}_{\mathrm{Cus}\rangle}\right\rangle \| R_{B}\left(\left\langle\operatorname{Cus}_{C u} \mid \mathcal{S}_{\mathrm{CUS}}\right\rangle\right)\right) \|\left(R_{\mathrm{I}}\left(\left\langle\mathrm{CLK}_{C l} \mid \mathcal{S}_{\mathrm{CLK}}\right\rangle\right) \| R_{\mathrm{II}}\left(\left\langle\mathrm{CLK}_{C l} \mid \mathcal{S}_{\mathrm{CLK}}\right\rangle\right)\right)\right.
$$

and the part of the labelled transition system of CCSP reachable from that process has $3 \times 3 \times 1 \times 1=9$ states and $6 \times 4=24$ transitions. 


\section{Petri nets}

A multiset over a set $S$ is a function $C: S \rightarrow \mathbb{N}$, i.e. $C \in \mathbb{N}^{S}$; let $|C|:=\sum_{x \in X} C(x)$; $x \in S$ is an element of $C$, notation $x \in C$, iff $C(x)>0$.

The function $\emptyset: S \rightarrow \mathbb{N}$, given by $\emptyset(x):=0$ for all $x \in S$, is the empty multiset over $S$. For multisets $C$ and $D$ over $S$ one writes $C \leq D$ iff $C(x) \leq D(x)$ for all $x \in S$;

$C \cap D$ denotes the multiset over $S$ with $(C \cap D)(x):=\min (C(x), D(x))$, $C+D$ denotes the multiset over $S$ with $(C+D)(x):=C(x)+D(x)$; and the multiset $C-D$ is only defined if $D \leq C$ and then $(C-D)(x):=C(x)-D(x)$. A multiset $C$ with $C(x) \leq 1$ for all $x$ is identified with the (plain) set $\{x \mid C(x)=1\}$. The construction $C:=\left\{f\left(x_{1}, \ldots, x_{n}\right) \mid x_{i} \in D_{i}\right\}$ of a set $C$ out of sets $D_{i}(i=1, \ldots, n)$ generalises naturally to multisets $C$ and $D_{i}$, taking the multiplicity $C(x)$ of an element $x$ to be $\sum_{f\left(x_{1}, \ldots, x_{n}\right)=x} D_{1}\left(x_{1}\right) \cdot \ldots \cdot D_{n}\left(x_{n}\right)$.

Definition 1. A (typed) Petri net is a tuple $N=\left(S, T, F, M_{0}, A, \ell\right)$ with

- $S$ and $T$ disjoint sets (of places and transitions),

- F : $((S \times T) \cup(T \times S)) \rightarrow \mathbb{N}$ (the flow relation including arc weights),

- $M_{0}: S \rightarrow \mathbb{N}$ (the initial marking),

- $A$ a set of actions, the type of the net, and

$-\ell: T \rightarrow A$ (the labelling function).

Petri nets are depicted by drawing the places as circles and the transitions as boxes, containing their label. Identities of places and transitions are displayed next to the net element. For $x, y \in S \cup T$ there are $F(x, y)$ arrows (arcs) from $x$ to $y$. When a Petri net represents a concurrent system, a global state of this system is given as a marking, a multiset $M$ of places, depicted by placing $M(s)$ dots (tokens) in each place $s$. The initial state is $M_{0}$.

The behaviour of a Petri net is defined by the possible moves between markings $M$ and $M^{\prime}$, which take place when a transition $t$ fires. In that case, $t$ consumes $F(s, t)$ tokens from each place $s$. Naturally, this can happen only if $M$ makes all these tokens available in the first place. Moreover, $t$ produces $F(t, s)$ tokens in each place $s$. Definition 2 formalises this notion of behaviour.

Definition 2. Let $N=\left(S, T, F, M_{0}, A, \ell\right)$ be a Petri net and $x \in S \cup T$. The multisets $\bullet x, x^{\bullet}: S \cup T \rightarrow \mathbb{N}$ are given by ${ }^{\bullet} x(y)=F(y, x)$ and $x^{\bullet}(y)=F(x, y)$ for all $y \in S \cup T$; for $t \in T$, the elements of $\bullet t$ and $t^{\bullet}$ are called pre- and postplaces of $t$, respectively. Transition $t \in T$ is enabled from the marking $M \in \mathbb{N}^{S}$-notation $M[t\rangle$-if $\bullet t \leq M$. In that case firing $t$ yields the marking $M^{\prime}:=M-{ }^{\bullet} t+t^{\bullet}$-notation $M[t\rangle M^{\prime}$.

A path $\pi$ of a Petri net $N$ is an alternating sequence $M_{0} t_{1} M_{1} t_{2} M_{2} t_{3} \ldots$ of markings and transitions, starting from the initial marking $M_{0}$ and either being infinite or ending in a marking $M_{n}$, such that $M_{k}\left[t_{k}\right\rangle M_{k+1}$ for all $k(<n)$. A marking is reachable if it occurs in such a path. The Petri net $N$ is safe if all reachable markings $M$ are plain sets, meaning that $M(s) \leq 1$ for all places $s$. It has bounded parallelism [16] if there is no reachable marking $M$ and infinite multiset of transitions $U$ such that $\sum_{t \in U} \bullet t \leq M$. In this paper I consider Petri nets with bounded parallelism only, and call them nets. 


\section{An operational Petri net semantics of CCSP}

This section recalls the operational Petri net semantics of CCSP, given by Olderog [26 27]. It associates a net $\llbracket P \rrbracket$ with each closed CCSP expression $P$.

The standard operational semantics of CCSP, presented in Section 2 yields one big labelled transition system for the entire language 1 Each individual closed CCSP expression $P$ appears as a state in this LTS. If desired, a process graph — an LTS enriched with an initial state-for $P$ can be extracted from this system-wide LTS by appointing $P$ as the initial state, and optionally deleting all states and transitions not reachable from $P$. In the same vein, an operational Petri net semantics yields one big Petri net for the entire language, but without an initial marking. I call such a Petri net unmarked. Each process $P \in \mathrm{T}_{\mathrm{CCSP}}$ corresponds with a marking $\operatorname{dex}(P)$ of that net. If desired, a Petri net $\llbracket P \rrbracket$ for $P$ can be extracted from this system-wide net by appointing $\operatorname{dex}(P)$ as its initial marking, taking the type of $\llbracket P \rrbracket$ to be $\alpha(P)$, and optionally deleting all places and transitions not reachable from $\operatorname{dex}(P)$.

The set $S_{\mathrm{CCSP}}$ of places in the net is the smallest set including:

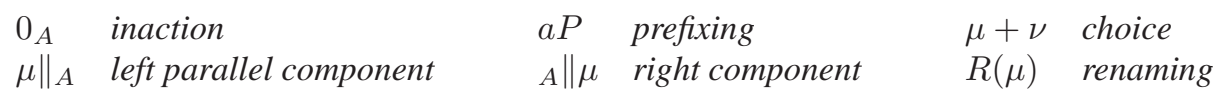

for $A \subseteq A c t, P \in \mathrm{T}_{\mathrm{CCSP}}, a \in A c t, \mu, \nu \in S_{\mathrm{CCSP}}$ and renamings $R$. The mapping dex $: \mathrm{T}_{\mathrm{CCSP}} \rightarrow \mathscr{P}\left(S_{\mathrm{CCSP}}\right)$ decomposing and expanding a process expression into a set of places is inductively defined by:

$$
\begin{array}{rlrl}
\operatorname{dex}(a P) & =\{a P\} & \operatorname{dex}(R(P)) & =R(\operatorname{dex}(P)) \\
\operatorname{dex}(P+Q) & =\operatorname{dex}(P)+\operatorname{dex}(Q) & \operatorname{dex}\left(\left\langle X_{A} \mid \mathcal{S}\right\rangle\right)=\operatorname{dex}\left(\left\langle\mathcal{S}_{X_{A}} \mid \mathcal{S}\right\rangle\right) \\
\operatorname{dex}(P \| Q) & =\operatorname{dex}(P)\left\|_{A} \cup{ }_{A}\right\| \operatorname{dex}(Q) \text { where } A=\alpha(P) \cap \alpha(Q) .
\end{array}
$$

Here $H\left\|_{A},{ }_{A}\right\| H, R(H)$ and $H+K$ for $H, K \subseteq S_{\mathrm{CCSP}}$ are defined element by element; e.g. $R(H)=\{R(\mu) \mid \mu \in H\}$. The binding matters, so that $\left({ }_{A} \| H\right)\left\|_{B} \neq{ }_{A}\right\|\left(H \|_{B}\right)$. Since I deal with guarded recursion only, dex is well-defined.

Following [27], I construct the unmarked Petri net $(S, T, F, A c t, \ell)$ of CCSP with $S:=S_{\mathrm{CCSP}}$, specifying the triple $(T, F, \ell)$ as a ternary relation $\rightarrow \subseteq \mathbb{N}^{S} \times A c t \times \mathbb{N}^{S}$. An element $H \stackrel{a}{\longrightarrow} J$ of this relation denotes a transition $t \in T$ with $\ell(t)=a$ such that $\bullet^{\bullet} t=H$ and $t^{\bullet}=J$. The transitions $H \stackrel{\alpha}{\longrightarrow} J$ are derived from the rules of Table 3 ,

Note that there is no rule for recursion. The transitions of a recursive process $\left\langle X_{A} \mid \mathcal{S}\right\rangle$ are taken care of indirectly by the decomposition $\operatorname{dex}\left(\left\langle X_{A} \mid \mathcal{S}\right\rangle\right)=\operatorname{dex}\left(\left\langle\mathcal{S}_{X_{A}} \mid \mathcal{S}\right\rangle\right)$, which expands the decomposition of a recursive call into a decomposition of an expression in which each recursive call is guarded by an action prefix.

Example 2. The Petri net semantics of the 2-customer 2-clerk store from Section 2 is displayed in Figure 4 It is more compact than the 9-state 24-transition labelled transition system. The name of the bottom-most place is ${ }_{S e r} \| \emptyset R_{\mathrm{II}}\left(\right.$ serve $\left.\left\langle\mathrm{CLK}_{C l} \mid \mathcal{S}_{\mathrm{CLK}}\right\rangle\right)$ where $S e r$ is the alphabet $\{$ I serves $A$, I serves $B$, II serves $A$, II serves $B\}$.

\footnotetext{
${ }^{1}$ A labelled transition system (LTS) is given by a set $S$ of states and a transition relation $T \subseteq S \times \mathscr{L} \times S$ for some set of labels $\mathscr{L}$. The LTS generated by CCSP has $S:=\mathrm{T}_{\mathrm{CCSP}}$, $\mathscr{L}:=$ Act and $T:=\rightarrow$.
} 
Table 3. Operational Petri net semantics of CCSP

\begin{tabular}{|c|c|}
\hline \multicolumn{2}{|c|}{$a P$}$\stackrel{a}{\longrightarrow} \operatorname{dex}(P)$ \\
\hline$H \stackrel{a}{\longrightarrow} J$ & $H \stackrel{a}{\longrightarrow} J$ \\
\hline$\overline{R(H) \stackrel{b}{\longrightarrow} R(J)}((a, b) \in R)$ & $\overline{H\left\|_{A} \stackrel{a}{\longrightarrow} J\right\|_{A}}(a \notin A)$ \\
\hline$H \bullet K \stackrel{a}{\longrightarrow} J$ & $H \stackrel{a}{\longrightarrow} J \quad K \stackrel{a}{\longrightarrow} L$ \\
\hline$\overline{H \cup(K+\operatorname{dex}(Q)) \stackrel{a}{\longrightarrow} J}$ & $\overline{H\left\|_{A} \cup \cup_{A}\right\| K \stackrel{a}{\longrightarrow} J\left\|_{A} \cup \cup_{A}\right\| L}$ \\
\hline$H \bullet K \stackrel{a}{\longrightarrow} J$ & $H \stackrel{a}{\longrightarrow} J$ \\
\hline$\overline{H \cup(\operatorname{dex}(P)+K) \stackrel{a}{\longrightarrow} J}$ & $\overline{A\left\|H \stackrel{a}{\longrightarrow}{ }_{A}\right\| J}$ \\
\hline
\end{tabular}

A progress assumption, as discussed in Section 1.4 disallows runs that stop after finitely many actions. So in each run some of the actions from Ser will occur infinitely often. When assuming strong fairness, each of those actions will occur infinitely often. When assuming only weak fairness, it is possible that II serves $A$ and II serves $B$ will never occur, as long as I serves $A$ and I serves $B$ each occur infinitely often, for in such a run the actions II serves $A$ and II serves $B$ are not enabled in every state (from some point onwards). However, it is not possible that I serves $B$ and II serves $B$ never occur, because in such a run, from some point onwards, the action I serves $B$ is enabled in every state.

When assuming justness but not weak fairness, a run that bypasses any two serving actions is possible, but a run that bypasses I serves $B$, II serves $A$ and II serves $B$ is excluded, because in such a run, from some point onwards, the action II serves $B$ is perpetually enabled, in the sense that both tokens in its preplaces never move away.

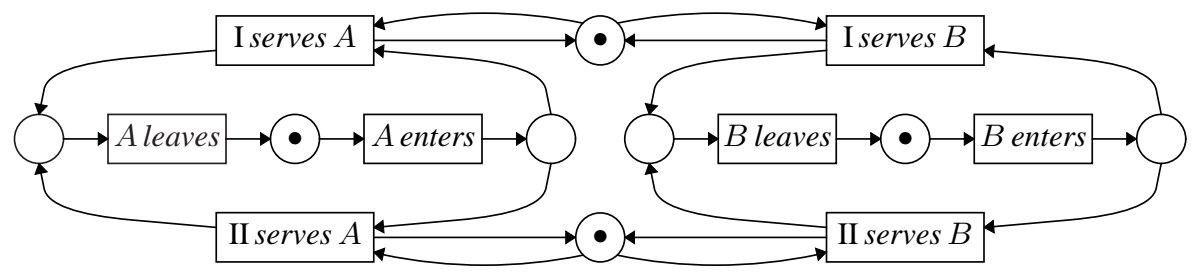

Fig. 4. Petri net semantics of the 2-customer 2-clerk store

Olderog [2627] shows that the Petri net $\llbracket P \rrbracket$ associated to a closed CCSP expression $P$ is safe, and that all its reachable markings are finite; the latter implies that it has bounded parallelism. The following result, from [26 27], shows that the standard interleaving semantics of CCSP is retrievable from the net semantics; it establishes a strong bisimulation relating any CCSP expression (seen as a state in a labelled transition system) with its interpretation as a marking in the Petri net of CCSP. 
Theorem 1. There exists a relation $\mathscr{B}$ between closed CCSP expressions and markings in the unmarked Petri net of CCSP, such that

- $P \mathscr{B} \operatorname{dex}(P)$ for each closed, well-typed CCSP expression with guarded recursion,

- if $P \mathscr{B} M$ and $P \stackrel{a}{\longrightarrow} P^{\prime}$ then there is a marking $M^{\prime}$ and transition $t$ with $\ell(t)=a$, $M[t\rangle M^{\prime}$ and $P \mathscr{B} M^{\prime}$, and

- if $P \mathscr{B} M$ and $M[t\rangle M^{\prime}$ then there is CCSP process $P^{\prime}$ with $P \stackrel{\ell(t)}{\longrightarrow} P^{\prime}$ and $P \mathscr{B} M^{\prime}$.

To formalise the concurrency requirement for his net semantics Olderog defines for each $n$-ary CCSP operator $o p$ an $n$-ary operation $o p_{\mathcal{N}}$ on safe Petri nets, inspired by proposals from [18[35|16], and requires that

$$
\begin{aligned}
\llbracket o p\left(P_{1}, \ldots, P_{n}\right) \rrbracket & \approx o p_{\mathcal{N}}\left(\llbracket P_{1} \rrbracket, \ldots, \llbracket P_{n} \rrbracket\right) \\
\llbracket\left\langle X_{A} \mid \mathcal{S}\right\rangle \rrbracket & \approx \llbracket\left\langle\mathcal{S}_{X_{A}} \mid \mathcal{S}\right\rangle \rrbracket
\end{aligned}
$$

for a suitable relation $\approx$. In fact, (2) turns out to hold taking for $\approx$ the identity relation. He establishes (1) taking for $\approx$ a relation he calls strong bisimilarity, whose definition will be recalled in Section 6 When a relation $\equiv$ includes $\approx$, and $(1)$ holds for $\approx$, then it also holds for $\equiv$.

The operations $o_{\mathcal{N}_{\mathcal{N}}}$ (i.e. $\left(0_{A}\right)_{\mathcal{N}}$ for $A \subseteq A c t, a_{\mathcal{N}}$ for $a \in A c t, R_{\mathcal{N}}$ for $R \subseteq A c t \times A c t$, $\|_{\mathcal{N}}$ and $+_{\mathcal{N}}$ ) are defined only up to isomorphism, but this is no problem as isomorphic nets are strongly bisimilar. The definition is recalled below-it generalises verbatim to non-safe nets, except that $+_{\mathcal{N}}$ is defined only for nets whose initial markings are nonempty plain sets.

Definition 3. [27] The net $0_{A}$ has type $A$ and consists of a single place, initially marked: $\left(0_{A}\right)_{\mathcal{N}}:=\left(\left\{0_{A}\right\}, \emptyset, \emptyset,\left\{0_{A}\right\}, A, \emptyset\right)$.

Given a net $N=(S, T, F, M, A, \ell)$ and $a \in A c t$, take $s_{0}, t_{a} \notin S \cup T$. Then the net $a_{\mathcal{N}} N$ is obtained from $N$ by the addition of the fresh place $s_{0}$ and the fresh transition $t_{a}$, labelled $a$, such that ${ }^{\bullet} t_{a}=\left\{s_{0}\right\}$ and $t_{a} \bullet=M$. The type of $a_{\mathcal{N}} N$ will be $A \cup\{a\}$ and the initial marking $\left\{s_{0}\right\}$.

Given a net $N=(S, T, F, M, A, \ell)$ and a renaming operator $R\left({ }_{-}\right)$, the net $R_{\mathcal{N}}(N)$ has type $R(A):=\{b \in A c t \mid \exists a \in A,(a, b) \in R\}$, the same places and initial marking as $N$, and transitions $t_{b}$ for each $t \in T$ and $b \in A c t$ with $(\ell(t), b) \in R$. One has ${ }^{\bullet} t_{b}:=\bullet$, $t_{b}{ }^{\bullet}:=t^{\bullet}$, and the label of $t_{b}$ will be $b$.

Given two nets $N_{i}=\left(S_{i}, T_{i}, F_{i}, M_{i}, A_{i}, \ell_{i}\right)(i=1,2)$, their parallel composition $N_{1} \|_{\mathcal{N}} N_{2}=(S, T, F, M, A, \ell)$ is obtained from the disjoint union of $N_{1}$ and $N_{2}$ by the omission of all transitions $t$ of $T_{1} \bullet T_{2}$ with $\ell(t) \in A_{1} \cap A_{2}$, and the addition of fresh transitions $\left(t_{1}, t_{2}\right)$ for all pairs $t_{i} \in T_{i}(i=1,2)$ with $\ell_{1}\left(t_{1}\right)=\ell_{2}\left(t_{2}\right) \in A_{1} \cap A_{2}$. Take $\bullet\left(t_{1}, t_{2}\right)={ }^{\bullet} t_{1}+{ }^{\bullet} t_{2},\left(t_{1}, t_{2}\right)^{\bullet}=t_{1} \bullet+t_{2} \bullet, \ell\left(t_{1}, t_{2}\right)=\ell\left(t_{1}\right)$, and $A:=A_{1} \cup A_{2}$.

Given nets $N_{i}=\left(S_{i}, T_{i}, F_{i}, M_{i}, A_{i}, \ell_{i}\right)$ with $M_{i} \neq \emptyset$ a plain set $(i=1,2)$, the net $N_{1}+\mathcal{N} N_{2}$ with type $A_{1} \cup A_{2}$ is obtained from the disjoint union of $N_{1}$ and $N_{2}$ by the addition of the set of fresh places $M_{1} \times M_{2}$-this set will be the initial marking of $N_{1}+{ }_{\mathcal{N}} N_{2}$ - and the addition of fresh transitions $t_{i}^{K}$ for any $t_{i} \in T_{i}$ and $\emptyset \neq K \leq \bullet t_{i} \cap M_{i}$. $\ell\left(t_{i}^{K}\right)=\ell_{i}(t),{ }^{\bullet} t_{1}^{K}={ }^{\bullet} t_{1}-K+\left(K \times M_{2}\right),{ }^{\bullet} t_{2}^{K}={ }^{\bullet} t_{2}-K+\left(M_{1} \times K\right)$ and $\left(t_{i}^{K}\right)^{\bullet}=t_{i} \bullet$. 


\section{Structure preserving bisimulation equivalence}

This section presents structure preserving bisimulation equivalence on nets.

Definition 4. Given two nets $N_{i}=\left(S_{i}, T_{i}, F_{i}, M_{i}, A_{i}, \ell_{i}\right)$, a link is a pair $\left(s_{1}, s_{2}\right) \in S_{1} \times S_{2}$ of places. A linking $l \in \mathbb{N}^{S_{1} \times S_{1}}$ is a multiset of links; it can be seen as a pair of markings with a bijection between them. Let $\pi_{i}(l) \in \mathbb{N}^{S_{i}}$ be these markings, given by $\pi_{1}(l)\left(s_{1}\right)=$ $\sum_{s_{2} \in S_{2}} l\left(s_{1}, s_{2}\right)$ for all $s_{1} \in S_{1}$ and $\pi_{2}(l)\left(s_{2}\right)=\sum_{s_{1} \in S_{1}} l\left(s_{1}, s_{2}\right)$ for all $s_{2} \in S_{2}$. A structure preserving bisimulation (sp-bisimulation) is a set $\mathscr{B}$ of linkings, such that

- if $c \leq l \in \mathscr{B}$ and $\pi_{1}(c)={ }^{\bullet} t_{1}$ for $t_{1} \in T_{1}$ then there are a transition $t_{2} \in T_{2}$ with $\ell\left(t_{2}\right)=\ell\left(t_{1}\right)$ and $\pi_{2}(c)={ }^{\bullet} t_{2}$, and a linking $\bar{c}$ such that $\pi_{1}(\bar{c})=t_{1} \bullet, \pi_{2}(\bar{c})=t_{2} \bullet$ and $\bar{l}:=l-c+\bar{c} \in \mathscr{B}$.

- if $c \leq l \in \mathscr{B}$ and $\pi_{2}(c)=\bullet t_{2}$ then there are a $t_{1}$ and a $\bar{c}$ with the same properties.

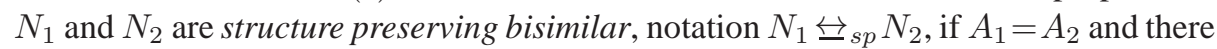
is a linking $l$ in a structure preserving bisimulation with $M_{1}=\pi_{1}(l)$ and $M_{2}=\pi_{2}(l)$.

Note that if $\mathscr{B}$ is an sp-bisimulation, then so is its downward closure $\{k \mid \exists l \in \mathscr{B} . k \leq l\}$. Moreover, if $\mathscr{B}$ is an sp-bisimulation between two nets, then the set of those linkings $l \in \mathscr{B}$ for which $\pi_{1}(l)$ and $\pi_{2}(l)$ are reachable markings is also an sp-bisimulation.

If $\mathcal{B}$ is a set of a links, let $\overline{\mathcal{B}}$ be the set of all linkings that are multisets over $\mathcal{B}$.

Proposition 1. Structure preserving bisimilarity is an equivalence relation.

Proof. The relation $\overline{I d}$, with $I d$ the identity relation on places, is an sp-bisimulation, showing that $N \overleftrightarrow{{ }_{s p}} N$ for any net $N$.

Given an sp-bisimulation $\mathscr{B}$, also $\left\{l^{-1} \mid l \in \mathscr{B}\right\}$ is an sp-bisimulation, showing

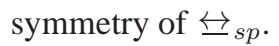

Given linkings $h \in \mathbb{N}^{S_{1} \times S_{3}}, k \in \mathbb{N}^{S_{1} \times S_{2}}$ and $l \in \mathbb{N}^{S_{2} \times S_{3}}$, write $h \in k ; l$ if there is a multiset $m \in \mathbb{N}^{S_{1} \times S_{2} \times S_{3}}$ of triples of places, with $k\left(s_{1}, s_{2}\right)=\sum_{s_{3} \in S} m\left(s_{1}, s_{2}, s_{3}\right)$, $l\left(s_{2}, s_{3}\right)=\sum_{s_{1} \in S} m\left(s_{1}, s_{2}, s_{3}\right)$ and $h\left(s_{1}, s_{3}\right)=\sum_{s_{2} \in S} m\left(s_{1}, s_{2}, s_{3}\right)$. Now, for spbisimulations $\mathscr{B}$ and $\mathscr{B}^{\prime}$, also $\mathscr{B} ; \mathscr{B}^{\prime}:=\left\{h \in k ; l \mid k \in \mathscr{B} \wedge l \in \mathscr{B}^{\prime}\right\}$ is an sp-bisimulation,

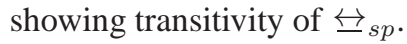

\section{Strong bisimilarity}

As discussed in the introduction and at the end of Section 4 , Olderog defined a relation of strong bisimilarity on safe Petri nets.

Definition 5. For $\mathcal{B} \subseteq S_{1} \times S_{2}$ a binary relation between the places of two safe nets $N_{i}=\left(S_{i}, T_{i}, F_{i}, M_{i}, A_{i}, \ell_{i}\right)$, write $\widehat{\mathcal{B}}$ for the set of all linkings $l \subseteq \mathcal{B}$ such that $\pi_{i}(l)$ is a reachable marking of $N_{i}$ for $i=1,2$ and $\mathcal{B} \cap\left(\pi_{1}(l) \times \pi_{2}(l)\right)=l$. Now a strong bisimulation as defined in [27] can be seen as a structure preserving bisimulation of the form $\widehat{\mathcal{B}}$. The nets $N_{1}$ and $N_{2}$ are strongly bisimilar if $A_{1}=A_{2}$ and there is a linking $l$ in a strong bisimulation with $M_{1}=\pi_{1}(l)$ and $M_{2}=\pi_{2}(l)$.

This reformulation of the definition from [27] makes immediately clear that strong bisimilarity of two safe Petri nets implies their structure preserving bisimilarity. Consequently, the concurrency requirement for the net semantics from Olderog, as formalised by Requirements (1) and (2) in Section 4 holds for structure preserving bisimilarity. 


\section{Compositionality}

In this section I show that structure preserving bisimilarity is a congruence for the op-

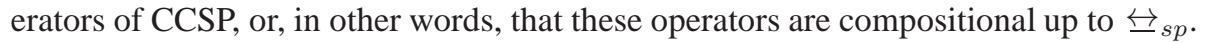

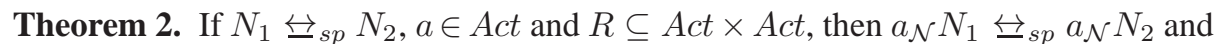

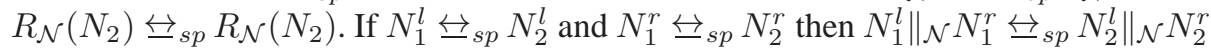

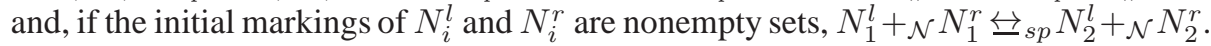

Proof. Let $N_{i}=\left(S_{i}, T_{i}, F_{i}, M_{i}, A_{i}, \ell_{i}\right)$ for $i=1,2$, and let $s_{i}$ and $u_{i}$ be the fresh place

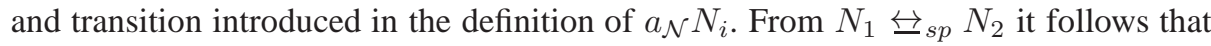
$A_{1}=A_{2}$ and hence $A_{1} \cup\{a\}=A_{2} \cup\{a\}$.

Let $\mathscr{B}$ be an sp-bisimulation containing a linking $k$ with $M_{i}=\pi_{i}(k)$ for $i=1,2$. Let $\mathscr{B}_{a}:=\mathscr{B} \cup\{h\}$, with $h=\left\{\left(s_{1}, s_{2}\right)\right\}$. Then $h$ links the initial markings of $a_{\mathcal{N}} N_{1}$ and $a_{\mathcal{N}} N_{2}$. Hence it suffices to show that $\mathscr{B}_{a}$ is an sp-bisimulation. So suppose $c \leq h$ and $\pi_{1}(c)=\bullet t_{1}$ for some $t_{1} \in T_{1}$. Then $c=h$ and $t_{1}=u_{1}$. Take $t_{2}:=u_{2}$ and $\bar{h}:=\bar{c}:=k$.

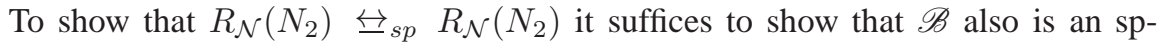
bisimulation between $R_{\mathcal{N}}\left(N_{2}\right)$ and $R_{\mathcal{N}}\left(N_{2}\right)$, which is straightforward.

Now let $N_{i}^{l}=\left(S_{i}^{l}, T_{i}^{l}, F_{i}^{l}, M_{i}^{l}, A_{i}^{l}, \ell_{i}^{l}\right)$ and $N_{i}^{r}=\left(S_{i}^{r}, T_{i}^{r}, F_{i}^{r}, M_{i}^{r}, A_{i}^{r}, \ell_{i}^{r}\right)$ for $i=1,2$. Let $A:=A_{1}^{l} \cap A_{1}^{r}=A_{2}^{l} \cap A_{2}^{r}$. Create the disjoint union of $N_{i}^{l}$ and $N_{i}^{r}$ in the definition of $N_{i}^{l} \|_{\mathcal{N}} N_{i}^{r}$ by renaming all places $s$ and transitions $t$ of $N_{i}^{l}$ into $s \|_{A}$ and $t \|_{A}$, and all places $s$ and transitions $t$ of $N_{i}^{r}$ into ${ }_{A} \| s$ and ${ }_{A} \| t$. Let $\mathscr{B}^{l}$ and $\mathscr{B}^{r}$ be sp-bisimulations containing linkings $k^{l}$ and $k^{r}$, respectively, with $M_{i}^{l}=\pi_{i}\left(k^{l}\right)$ and $M_{i}^{r}=\pi_{i}\left(k^{r}\right)$, for $i=1,2$. Take $\mathscr{B}:=\left\{\left(h^{l} \|_{A}\right)+\left({ }_{A} \| h^{r}\right) \mid h^{l} \in \mathscr{B}^{l} \wedge h^{r} \in \mathscr{B}^{r}\right\}$, where $h^{l} \|_{A}:=\left\{\left(s_{1}\left\|_{A}, s_{2}\right\|_{A}\right)\right.$ $\left.\mid\left(s_{1}, s_{2}\right) \in h^{l}\right\}$, and $A \| h^{r}$ is defined likewise. Then $\pi_{i}\left(\left(k^{l} \|_{A}\right)+\left({ }_{A} \| k^{r}\right)\right)=\pi_{i}\left(k^{l}\right) \|_{A}+$ ${ }_{A}\left\|\pi_{i}\left(k^{r}\right)=M_{i}^{l}\right\|_{A}+{ }_{A} \| M_{i}^{r}$ is the initial marking of $N_{i}^{l} \|_{\mathcal{N}} N_{i}^{r}$ for $i=1,2$, so it suffices to show that $\mathscr{B}$ is an sp-bisimulation.

So suppose $c \leq\left(h^{l} \|_{A}\right)+\left({ }_{A} \| h^{r}\right) \in \mathscr{B}$ with $h^{l} \in \mathscr{B}^{l} \wedge h^{r} \in \mathscr{B}^{r}$ and $\pi_{1}(c)={ }^{\bullet} t_{1}$ for $t_{1}$ a transition of $N_{1}^{l} \|_{\mathcal{N}} N_{1}^{r}$. Then $c$ has the form $\left(c^{l} \|_{A}\right)+\left({ }_{A} \| c^{r}\right)$ for $c^{l} \leq h^{l} \in \mathscr{B}^{l}$ and $c^{r} \leq h^{r} \in \mathscr{B}^{r}$, and $t_{1}$ has the form (i) $t_{1}^{l} \|_{A}$ for $t_{1}^{l} \in T_{1}^{l}$ with $\ell_{1}^{l}\left(t_{1}^{l}\right) \notin A$, or (ii) $\left(t_{1}^{l}\left\|_{A},{ }_{A}\right\| t_{1}^{r}\right)$ for $t_{1}^{l} \in T_{1}^{l}$ and $t_{1}^{r} \in T_{1}^{r}$ with $\ell_{1}^{l}\left(t_{1}^{l}\right)=\ell_{1}^{r}\left(t_{1}^{r}\right) \in A$, or (iii) $A \| t_{1}^{r}$ for $t_{1}^{r} \in T_{1}^{r}$ with $\ell_{1}^{r}\left(t_{1}^{r}\right) \notin A$. In case (i) one has $c^{r}=\emptyset$ and $\pi_{1}\left(c^{l}\right)={ }^{\bullet} t_{1}^{l}$, whereas in case (ii) $\pi_{1}\left(c^{l}\right)={ }^{\bullet} t_{1}^{l}$ and $\pi_{1}\left(c^{r}\right)={ }^{\bullet} t_{1}^{r}$. I only elaborate case (ii); the other two proceed likewise. Since $\mathscr{B}^{l}$ is an sp-bisimulation, there are a transition $t_{2}^{l}$ with $\ell_{2}^{l}\left(t_{2}^{l}\right)=\ell_{1}^{l}\left(t_{1}^{l}\right)$ and $\pi_{2}\left(c^{l}\right)={ }^{\bullet} t_{2}^{l}$, and a linking $\bar{c}^{l}$ such that $\pi_{1}\left(\bar{c}^{l}\right)=t_{1}^{l^{\bullet}}, \pi_{2}\left(\bar{c}^{l}\right)=t_{2}^{l}$ and $\bar{h}^{l}:=h^{l}-c^{l}+\bar{c}^{l} \in \mathscr{B}^{l}$. Likewise, since $\mathscr{B}^{r}$ is an sp-bisimulation, there are a transition $t_{2}^{r}$ with $\ell_{2}^{r}\left(t_{2}^{r}\right)=\ell_{1}^{r}\left(t_{1}^{r}\right)$ and $\pi_{2}\left(c^{r}\right)=\bullet t_{2}^{r}$, and a linking $\bar{c}^{r}$ such that $\pi_{1}\left(\bar{c}^{r}\right)=t_{1}^{r \bullet}, \pi_{2}\left(\bar{c}^{r}\right)=t_{2}^{r \bullet}$ and $\bar{h}^{r}:=$ $h^{r}-c^{r}+\bar{c}^{r} \in \mathscr{B}^{r}$. Take $t_{2}:=\left(t_{2}^{l}\left\|_{A},{ }_{A}\right\| t_{2}^{r}\right)$. This transition has the same label as $t_{2}^{l}, t_{2}^{r}, t_{1}^{l}, t_{1}^{r}$ and $\left(t_{1}^{l}\left\|_{A},{ }_{A}\right\| t_{1}^{r}\right)=t_{1}$. Moreover, $\pi_{2}(c)=\pi_{2}\left(c^{l}\right)\left\|_{A}+{ }_{A}\right\| \pi_{2}\left(c^{r}\right)=$ ${ }^{\circ} t_{2}^{l}\left\|_{A}+{ }_{A}\right\|^{\bullet} t_{2}^{r}={ }^{\bullet} t_{2}$. Take $\bar{c}:=\left(\bar{c}^{l} \|_{A}\right)+\left({ }_{A} \| \bar{c}^{r}\right)$. Then $\pi_{1}(\bar{c})=t_{1} \bullet, \pi_{2}(\bar{c})=t_{2} \bullet$ and $\bar{h}:=\left(h^{l} \|_{A}\right)+\left({ }_{A} \| h^{r}\right)-c+\bar{c}=\left(\bar{h}^{l} \|_{A}\right)+\left({ }_{A} \| \bar{h}^{r}\right) \in \mathscr{B}$.

Let $N_{i}^{l}=\left(S_{i}^{l}, T_{i}^{l}, F_{i}^{l}, M_{i}^{l}, A_{i}^{l}, \ell_{i}^{l}\right)$ and $N_{i}^{r}=\left(S_{i}^{r}, T_{i}^{r}, F_{i}^{r}, M_{i}^{r}, A_{i}^{r}, \ell_{i}^{r}\right)$ for $i=1,2$, with $M_{i}^{l}$ and $M_{i}^{r}$ nonempty plain sets, but this time I assume the nets to already be disjoint, and such that all the places and transitions added in the construction of $N_{i}^{l}+\mathcal{N} N_{i}^{r}$ are fresh. Let $\mathscr{B}^{l}$ and $\mathscr{B}^{r}$ be as above. Without loss of generality I may assume that the linkings $h$ in $\mathscr{B}^{l}$ and $\mathscr{B}^{r}$ have the property that $\pi_{i}(h)$ is a reachable marking for 
$i=1,2$, so that the restriction of $\pi_{i}(h)$ to $M_{i}^{l}$ or $M_{i}^{r}$ is a plain set. Define

$$
\begin{aligned}
\mathscr{B}^{+}:= & \left\{h_{\bullet}^{l}+\left(h_{+}^{l} \otimes k^{r}\right) \mid h_{\bullet}^{l}+h_{+}^{l} \in \mathscr{B}^{l} \wedge h_{+}^{l} \lesseqgtr k^{l}\right\} \\
& \left\{h_{\bullet}^{r}+\left(k^{l} \otimes h_{+}^{r}\right) \mid h_{\bullet}^{r}+h_{+}^{r} \in \mathscr{B}^{r} \wedge h_{+}^{r} \nRightarrow k^{r}\right\} \cup\left\{k^{l} \otimes k^{r}\right\}
\end{aligned}
$$

where $h^{l} \otimes h^{r}:=\left\{\left(\left(s_{1}^{l}, s_{1}^{r}\right),\left(s_{2}^{l}, s_{2}^{r}\right)\right) \mid\left(s_{1}^{l}, s_{2}^{l}\right) \in h^{l} \wedge\left(s_{1}^{r}, s_{2}^{r}\right) \in h^{r}\right\}$. Now $\pi_{i}\left(k^{l} \otimes k^{r}\right)=$ $\pi_{i}\left(k^{l}\right) \times \pi_{i}\left(k^{r}\right)=M_{i}^{l} \times M_{i}^{r}$ is the initial marking of $N_{i}^{l}+\mathcal{N} N_{i}^{r}$, so again it suffices to show that $\mathscr{B}^{+}$is an sp-bisimulation.

So suppose $c \leq h_{\bullet}^{l}+\left(h_{+}^{l} \otimes k^{r}\right) \in \mathscr{B}^{+}$with $h_{\bullet}^{l}+h_{+}^{l} \in \mathscr{B}^{l}, h_{+}^{l} \varsubsetneqq k^{l}$ and $\pi_{1}(c)={ }^{\bullet} t_{1}$ for $t_{1}$ a transition of $N_{1}^{l}+\mathcal{N} N_{1}^{r}$.

First consider the case that $c \leq h_{\bullet}^{l}$. Then $c \leq h_{\bullet}^{l} \leq h_{\bullet}^{l}+h_{+}^{l} \in \mathscr{B}^{l}$. Since $\mathscr{B}^{l}$ is an sp-bisimulation, there are a transition $t_{2} \in T_{2}^{l}$ with $\ell_{2}^{l}\left(t_{2}\right)=\ell_{1}^{l}\left(t_{1}\right)$ and $\pi_{2}(c)={ }^{\bullet} t_{2}$, and a linking $\bar{c}$ such that $\pi_{1}(\bar{c})=t_{1}^{\bullet}, \pi_{2}(\bar{c})=t_{2}^{\bullet}$ and $h_{\bullet}^{l}+h_{+}^{r}-c+\bar{c} \in \mathscr{B}^{l}$. Now $h_{\bullet}^{l}+\left(h_{+}^{l} \otimes k^{r}\right)-c+\bar{c}=\left(h_{\bullet}^{l}-c+\bar{c}\right)+\left(h_{+}^{r} \otimes k_{2}\right) \in \mathscr{B}^{+}$because $\left(h_{\bullet}^{l}-c+\bar{c}\right)+h_{+}^{r} \in \mathscr{B}^{l}$.

In the remaining case $\pi_{1}(c)$ contains a place $\left(s_{1}^{l}, s_{1}^{r}\right) \in M_{1}^{l} \times M_{1}^{r}$, so $t_{1}$ must have either the form $t_{1 l}^{K}$ with $\emptyset \neq K \leq{ }^{\bullet} t_{1}^{l} \cap M_{1}^{l}$ for some $t_{1}^{l} \in T_{1}^{l}$, or $t_{1 r}^{K}$ with $\emptyset \neq K \leq$ ' $t_{1}^{r} \cap M_{1}^{r}$ for some $t_{1}^{r} \in T_{1}^{r}$. First assume, towards a contradiction, that $t_{1}=t_{1 r}^{K}$. Then $M_{1}^{l} \times K \leq t_{1 r}^{K}=\pi_{1}(c) \leq \pi_{1}\left(h_{\bullet}^{l}\right)+\pi_{1}\left(h_{+}^{l} \otimes k^{r}\right)$. Since the places in $M_{1}^{l} \times K \subseteq M_{1}^{l} \times M_{1}^{r}$ are fresh, it follows that $M_{1}^{l} \times K \leq \pi_{1}\left(h_{+}^{l} \otimes k^{r}\right) \leq \pi_{1}\left(h_{+}^{l}\right) \times \pi_{1}\left(k^{r}\right) \leq \pi_{1}\left(h_{+}^{l}\right) \times M_{1}^{r}$, implying that $M_{1}^{l} \leq \pi_{1}\left(h_{+}^{l}\right)$ and $K \leq M_{1}^{r}$-here I use that $M_{1}^{l} \neq \emptyset \neq K$ and $\pi_{1}\left(h_{+}^{l}\right)$ and $M_{1}^{r}$ are plain sets. However, the condition $h_{+}^{l} \lesseqgtr k^{l}$ implies that $\pi_{1}\left(h_{+}^{l}\right) \varsubsetneqq \pi_{1}\left(k^{l}\right)=M_{1}^{l}$, yielding a contradiction. Hence $t_{1}$ is of the form $t_{1 l}^{K}$.

Since $\pi_{1}(c)={ }^{\bullet} t_{1 l}^{K}={ }^{\bullet} t_{1}^{l}-K+\left(K \times M_{1}^{r}\right)$, the linking $c$ must have the form $c_{\bullet}+c^{\prime}$ with $\pi_{1}\left(c_{\bullet}\right)={ }^{\bullet} t_{1}^{l}-K$ and $\pi_{1}\left(c^{\prime}\right)=K \times M_{1}^{r}$. As no place in ${ }^{\bullet} t_{1}^{l}-K$ can be in $M_{1}^{l} \times M_{1}^{r} \supseteq \pi_{1}\left(h_{+}^{l} \otimes k^{r}\right)$, it follows that $c_{\bullet} \leq h_{\bullet}^{l}$. Likewise, as none of the places in $K \times M_{1}^{r}$ can be in $\pi_{1}\left(h_{\bullet}^{l}\right)$, it follows that $c^{\prime} \leq h_{+}^{l} \otimes k^{r}$. Thus $K \times M_{1}^{r}=\pi_{1}\left(c^{\prime}\right) \leq$ $\pi_{1}\left(h_{+}^{l} \otimes k^{r}\right) \leq \pi_{1}\left(h_{+}^{l}\right) \times \pi_{1}\left(k^{r}\right) \leq \pi_{1}\left(h_{+}^{l}\right) \times M_{1}^{r}$, implying $K \leq \pi_{1}\left(h_{+}^{l}\right)$ - again using that $\pi_{1}\left(h_{+}^{l}\right)$ and $M_{1}^{r} \neq \emptyset$ are plain sets. The linking $h_{+}^{l} \otimes k^{r}$ has the property that its projection $\pi_{1}\left(h_{+}^{l} \otimes k^{r}\right)$ is a plain set. Since a subset $c^{\prime \prime}$ of a such linking is completely determined by its first projection $\pi_{1}\left(c^{\prime \prime}\right)$, it follows that $c^{\prime}=c_{+} \otimes k^{r}$ for the unique linking $c_{+} \leq h_{+}^{l}$ with $\pi_{1}\left(c_{+}\right)=K$.

Now $c_{\bullet}+c_{+} \leq h_{\bullet}^{l}+h_{+}^{l} \in \mathscr{B}^{l}$ and $\pi_{1}\left(c_{\bullet}+c_{+}\right)=\left({ }^{\bullet} t_{1}^{l}-K\right)+K={ }^{\bullet} t_{1}^{l}$. Since $\mathscr{B}^{l}$ is an sp-bisimulation, there are a transition $t_{2}^{l} \in T_{2}^{l}$ with $\ell_{2}^{l}\left(t_{2}^{l}\right)=\ell_{1}^{l}\left(t_{1}^{l}\right)$ and $\pi_{2}\left(c_{\bullet}+c_{+}\right)={ }^{\bullet} t_{2}^{l}$, and a linking $\bar{c}$ such that $\pi_{1}(\bar{c})=t_{1}^{\bullet}, \pi_{2}(\bar{c})=t_{2}^{\bullet}$ and $h_{\bullet}^{l}+h_{+}^{l}-\left(c_{\bullet}+c_{+}\right)+\bar{c} \in \mathscr{B}^{l}$. Let $L:=\pi_{2}\left(c_{+}\right)$. Then $L \neq \emptyset$ since $K \neq \emptyset, L=\pi_{2}\left(c_{+}\right) \leq \pi_{2}\left(h_{+}^{l}\right) \leq \pi_{2}\left(k^{l}\right)=M_{2}^{l}$ and $L=\pi_{2}\left(c_{+}\right) \leq \pi_{2}\left(c_{\bullet}+c_{+}\right)={ }^{\bullet} t_{2}^{l}$. By Definition $3 N_{2}^{l}+{ }_{\mathcal{N}} N_{2}^{r}$ has a transition $t_{2 l}^{L}$ with $\ell\left(t_{2 l}^{L}\right)=\ell_{2}^{l}\left(t_{2}^{l}\right)=\ell_{1}^{l}\left(t_{1}^{l}\right)=\ell\left(t_{1 l}^{L}\right),{ }^{\bullet} t_{2 l}^{L}=\bullet t_{2}^{l}-L+\left(L \times M_{2}^{l}\right)=\pi_{2}\left(c_{\bullet}+c_{+}\right)-\pi_{2}\left(c_{+}\right)+$ $\left(\pi_{2}\left(c_{+}\right) \times \pi_{1}\left(k^{r}\right)\right)=\pi_{2}\left(c_{\bullet}+\left(c_{+} \otimes k^{r}\right)\right)=\pi_{2}(c)$ and $t_{2 l}^{L} \bullet=t_{2}^{\bullet}=\pi_{2}(\bar{c})$. Moreover, $\pi_{1}(\bar{c})=t_{1}^{\bullet}=t_{1}^{K^{\bullet}}$. Finally, $h_{\bullet}^{l}+\left(h_{+}^{l} \otimes k^{r}\right)-c+\bar{c}=\left(h_{\bullet}^{l}-c_{\bullet}+\bar{c}\right)+\left(\left(h_{+}^{l}-c_{+}\right) \otimes k^{r}\right) \in \mathscr{B}^{+}$ since $\left(h_{\bullet}^{l}-c_{\bullet}+c^{\prime}\right)+\left(h_{+}^{l}-c_{+}\right) \in \mathscr{B}^{l}$ and $h_{+}^{l}-c_{+} \leq h_{+}^{l} \lesseqgtr k^{l}$.

The case supposing $c \leq h_{\bullet}^{r}+\left(k^{r} \otimes h_{+}^{r}\right) \in \mathscr{B}^{+}$follows by symmetry, whereas the case $c \leq k^{l} \otimes k^{r}$ proceeds by simplification of the other two cases. 


\section{Processes of nets and causal equivalence}

A process of a net $N$ [29|9]19] is essentially a conflict-free, acyclic net together with a mapping function to $N$. It can be obtained by unwinding $N$, choosing one of the alternatives in case of conflict. It models a run, or concurrent computation, of $N$. The acyclic nature of the process gives rise to a notion of causality for transition firings in the original net via the mapping function. A conflict present in the original net is represented by the existence of multiple processes, each representing one possible way to decide the conflict. This notion of process differs from the one used in process algebra; there a "process" refers to the entire behaviour of a system, including all its choices.

Definition 6. A causal ne $\mathbb{Z}^{2}$ is a net $\mathcal{N}=\left(\mathcal{S}, \mathcal{T}, \mathcal{F}, \mathcal{M}_{0}, \mathcal{A}, \ell_{\mathcal{N}}\right)$ satisfying

- $\forall s \in \mathcal{S} .\left|{ }^{\bullet} s\right| \leq 1 \geq\left|s^{\bullet}\right| \wedge \mathcal{M}_{0}(s)= \begin{cases}1 & \text { if } \bullet s=\emptyset \\ 0 & \text { otherwise, }\end{cases}$

- $\mathcal{F}$ is acyclic, i.e., $\forall x \in \mathcal{S} \cup \mathcal{T}$. $(x, x) \notin \mathcal{F}^{+}$, where $\mathcal{F}^{+}$is the transitive closure of $\{(x, y) \mid \mathcal{F}(x, y)>0\}$,

- and $\left\{t \in \mathcal{T} \mid(t, u) \in \mathcal{F}^{+}\right\}$is finite for all $u \in \mathcal{T}$.

A folding from a net $\mathcal{N}=\left(\mathcal{S}, \mathcal{T}, \mathcal{F}, \mathcal{M}_{0}, \mathcal{A}, \ell_{\mathcal{N}}\right)$ into a net $N=\left(S, T, F, M_{0}, A, \ell\right)$ is a function $\rho: \mathcal{S} \cup \mathcal{T} \rightarrow S \cup T$ with $\rho(\mathcal{S}) \subseteq S$ and $\rho(\mathcal{T}) \subseteq T$, satisfying

- $\mathcal{A}=A$ and $\ell_{\mathcal{N}}(t)=\ell(\rho(t))$ for all $t \in \mathcal{T}$,

- $\rho\left(\mathcal{M}_{0}\right)=M_{0}$, i.e. $M_{0}(s)=\left|\rho^{-1}(s) \cap \mathcal{M}_{0}\right|$ for all $s \in S$, and

- $\forall t \in \mathcal{T}, s \in S . F(s, \rho(t))=\left|\rho^{-1}(s) \cap \bullet t\right| \wedge F(\rho(t), s)=\left|\rho^{-1}(s) \cap t^{\bullet}\right| .3$

A pair $\mathcal{P}=(\mathcal{N}, \rho)$ of a causal net $\mathcal{N}$ and a folding of $\mathcal{N}$ into a net $N$ is a process of $N$. $\mathcal{P}$ is called finite if $\mathcal{T}$ is finite.

Note that if $N$ has bounded parallelism, than so do all of its processes.

Definition 7. [27] A net $\mathcal{N}$ is called a causal net of a net $N$ if it is the first component of a process $(\mathcal{N}, \rho)$ of $N$. Two nets $N_{1}$ and $N_{2}$ are causal equivalent, notation $\equiv_{\text {caus }}$, if they have the same causal nets.

Olderog shows that his relation of strong bisimilarity is included in $\equiv_{\text {caus }}$ [27], and thereby establishes the concurrency requirement (1) from Section 4 for $\equiv_{\text {caus }}$.

For $\mathcal{N}=\left(\mathcal{S}, \mathcal{T}, \mathcal{F}, \mathcal{M}_{0}, \mathcal{A}, \ell_{\mathcal{N}}\right)$ a causal net, let $\mathcal{N}^{\circ}:=\left\{s \in \mathcal{S} \mid s^{\bullet}=\emptyset\right\}$. The following result supports the claim that finite processes model finite runs.

Proposition 2. [19, Theorems 3.5 and 3.6] $M$ is a reachable marking of a net $N$ iff $N$ has a finite process $(\mathcal{N}, \rho)$ with $\rho\left(\mathcal{N}^{\circ}\right)=M$. Here $\rho\left(\mathcal{N}^{\circ}\right)(s)=\left|\rho^{-1}(s) \cap \mathcal{N}^{\circ}\right|$.

\footnotetext{
${ }^{2}$ A causal net [29]34] is traditionally called an occurrence net [9]19]33]. Here, following [27], I will not use the terminology "occurrence net" in order to avoid confusion with the occurrence nets of [25|36]; the latter extend causal nets with forward branching places, thereby capturing all runs of the represented system, together with the branching structure between them.

${ }^{3}$ For $H \subseteq \mathcal{S}$, the multiset $\rho(H) \in \mathbb{N}^{S}$ is defined by $\rho(H)(s)=\left|\rho^{-1}(s) \cap H\right|$. Using this, these conditions can be reformulated as $\rho\left({ }^{\bullet} t\right)={ }^{\bullet} \rho(t)$ and $\rho\left(t^{\bullet}\right)=\rho(t)^{\bullet}$.
} 
A process is not required to represent a completed run of the original net. It might just as well stop early. In those cases, some set of transitions can be added to the process such that another (larger) process is obtained. This corresponds to the system taking some more steps and gives rise to a natural order between processes.

Definition 8. Let $\mathcal{P}=\left(\left(\mathcal{S}, \mathcal{T}, \mathcal{F}, \mathcal{M}_{0}, \mathcal{A}, \ell\right), \rho\right)$ and $\mathcal{P}^{\prime}=\left(\left(\mathcal{S}^{\prime}, \mathcal{T}^{\prime}, \mathcal{F}^{\prime}, \mathcal{M}_{0}^{\prime}, \mathcal{A}^{\prime}, \ell^{\prime}\right), \rho^{\prime}\right)$ be two processes of the same net. $\mathcal{P}^{\prime}$ is a prefix of $\mathcal{P}$, notation $\mathcal{P}^{\prime} \leq \mathcal{P}$, and $\mathcal{P}$ an extension of $\mathcal{P}^{\prime}$, iff $\mathcal{S}^{\prime} \subseteq \mathcal{S}, \mathcal{T}^{\prime} \subseteq \mathcal{T}, \mathcal{M}_{0}^{\prime}=\mathcal{M}_{0}, \mathcal{F}^{\prime}=\mathcal{F} \uparrow\left(\mathcal{S}^{\prime} \times \mathcal{T}^{\prime} \cup \mathcal{T}^{\prime} \times \mathcal{S}^{\prime}\right)$ and $\rho^{\prime}=\rho \uparrow\left(\mathcal{S}^{\prime} \cup \mathcal{T}^{\prime}\right)$. (This implies that $\mathcal{A}^{\prime}=\mathcal{A}$ and $\ell^{\prime}=\ell \uparrow \mathcal{T}$.)

The requirements above imply that if $\mathcal{P}^{\prime} \leq \mathcal{P},(x, y) \in \mathcal{F}^{+}$and $y \in \mathcal{S}^{\prime} \cup \mathcal{T}^{\prime}$ then $x \in$ $\mathcal{S}^{\prime} \cup \mathcal{T}^{\prime}$. Conversely, any subset $\mathcal{T}^{\prime} \subseteq \mathcal{T}$ satisfying $(t, u) \in \mathcal{F}^{+} \wedge u \in \mathcal{T}^{\prime} \Rightarrow t \in \mathcal{T}^{\prime}$ uniquely determines a prefix of $\mathcal{P}$. A process $(\mathcal{N}, \rho)$ of a net $N$ is initial if $\mathcal{N}$ contains no transitions; then $\rho\left(\mathcal{N}^{\circ}\right)$ is the initial marking of $N$. Any process has an initial prefix.

Proposition 3. [19, Theorem 3.17] If $\mathcal{P}_{i}=\left(\left(\mathcal{S}_{i}, \mathcal{T}_{i}, \mathcal{F}_{i}, \mathcal{M}_{0 i}, \mathcal{A}_{i}, \ell_{i}\right), \rho_{i}\right)(i \in \mathbb{N})$ is a chain of processes of a net $N$, satisfying $\mathcal{P}_{i} \leq \mathcal{P}_{j}$ for $i \leq j$, then there exists a unique process $\mathcal{P}=\left(\left(\mathcal{S}, \mathcal{T}, \mathcal{F}, \mathcal{M}_{0}, \mathcal{A}, \ell\right), \rho\right)$ of $N$ with $\mathcal{S}=\bigcup_{i \in \mathbb{N}} \mathcal{S}_{i}$ and $\mathcal{T}=\bigcup_{i \in \mathbb{N}} \mathcal{T}_{i}$ - the limit of this chain-such that $\mathcal{P}_{i} \leq \mathcal{P}$ for all $i \in \mathbb{N}$.

In [29 9 19] processes were defined without the third requirement of Definition 6 Goltz and Reisig [19] observed that certain processes did not correspond with runs of systems, and proposed to restrict the notion of a process to those that can be obtained as the limit of a chain of finite processes [19, end of Section 3]. By [19, Theorems 3.18 and 2.14], for processes of finite nets this limitation is equivalent with imposing the third bullet point of Definition 6. My restriction to nets with bounded parallelism serves to recreate this result for processes of infinite nets.

Proposition 4. Any process of a net can be obtained as the limit of a chain of finite approximations.

Proof. Define the depth of a transition $u$ in a causal net as one more than the maximum of the depth of all transitions $t$ with $t F^{+} u$. Since the set of such transitions $t$ is finite, the depth of a transition $u$ is a finite integer. Now, given a process $\mathcal{P}$, the approximation $\mathcal{P}_{i}$ is obtained by restricting to those transitions in $\mathcal{P}$ of depth $\leq i$, together with all their pre- and postplaces, and keeping the initial marking. Clearly, these approximations form a chain, with limit $\mathcal{P}$. By induction on $i$ one shows that $\mathcal{P}_{i}$ is finite. For $\mathcal{P}_{0}$ this is trivial, as it has no transitions. Now assume $\mathcal{P}_{i}$ is finite but $\mathcal{P}_{i+1}$ is not. Executing, in $\mathcal{P}_{i+1}$, all transitions of $\mathcal{P}_{i}$ one by one leads to a marking of $\mathcal{P}_{i+1}$ in which all remaining transitions of $\mathcal{P}_{i+1}$ are enabled. As these transitions cannot have common preplaces, this violates the assumption that $\mathcal{P}_{i+1}$ has bounded parallelism.

\section{A process-based characterisation of sp-bisimilarity}

This section presents an alternative characterisation of sp-bisimilarity that will be instru-

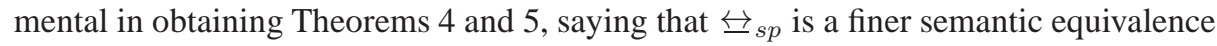
than $\equiv_{\text {caus }}$ and $\approx_{h}$. This characterisation could have been presented as the original def-

inition; however, the latter is instrumental in showing that $\overleftrightarrow{C}_{s p}$ is coarser than $\approx_{p b}$ and $\equiv_{o c c}$, and implied by Olderog's strong bisimilarity. 
Definition 9. A process-based sp-bisimulation between two nets $N_{1}$ and $N_{2}$ is a set $\mathscr{R}$ of triples $\left(\rho_{1}, \mathcal{N}, \rho_{2}\right)$ with $\left(\mathcal{N}, \rho_{i}\right)$ a finite process of $N_{i}$, for $i=1,2$, such that

- $\mathscr{R}$ contains a triple $\left(\rho_{1}, \mathcal{N}, \rho_{2}\right)$ with $\mathcal{N}$ a causal net containing no transitions,

- if $\left(\rho_{1}, \mathcal{N}, \rho_{2}\right) \in \mathscr{R}$ and $\left(\mathcal{N}^{\prime}, \rho_{i}^{\prime}\right)$ with $i \in\{1,2\}$ is a fin. proc. of $N_{i}$ extending $\left(\mathcal{N}, \rho_{i}\right)$ then $N_{j}$ with $j:=3-i$ has a process $\left(\mathcal{N}^{\prime}, \rho_{j}^{\prime}\right) \geq\left(\mathcal{N}, \rho_{j}\right)$ such that $\left(\rho_{1}^{\prime}, \mathcal{N}^{\prime}, \rho_{2}^{\prime}\right) \in \mathscr{R}$.

Theorem 3. Two nets are sp-bisimilar iff there exists a process-based sp-bisimulation between them.

Proof. Let $\mathscr{R}$ be a process-based sp-bisimulation between nets $N_{1}$ and $N_{2}$. Define $\mathscr{B}:=\left\{\left\{\left(\rho_{1}(\mathfrak{s}), \rho_{2}(\mathfrak{s})\right) \mid \mathfrak{s} \in \mathcal{N}^{\circ}\right\} \mid\left(\rho_{1}, \mathcal{N}, \rho_{2}\right) \in \mathscr{R}\right\}$. Then $\mathscr{B}$ is an sp-bisimulation:

- Let $c \leq l \in \mathscr{B}$ and $\pi_{1}(c)={ }^{\bullet} t_{1}$ for $t_{1} \in T_{1}$. Then $l=\left\{\left(\rho_{1}(\mathfrak{s}), \rho_{2}(\mathfrak{s}) \mid \mathfrak{s} \in \mathcal{N}^{\circ}\right\}\right.$ for some $\left(\rho_{1}, \mathcal{N}, \rho_{2}\right) \in \mathscr{R}$. Extend $\mathcal{N}$ to $\mathcal{N}^{\prime}$ by adding a fresh transition $\mathfrak{t}$ and fresh places $s_{i}$ for $s \in S_{1}$ and $i \in \mathbb{N}$ with $F_{1}\left(t_{1}, s\right)>i$; let $\bullet \mathfrak{t}=\left\{\mathfrak{s} \in \mathcal{N}^{\circ} \mid \rho_{1}(\mathfrak{s}) \in \bullet^{\bullet} t_{1}\right\}$ and $\mathfrak{t}^{\bullet}=\left\{s_{i} \mid s \in S_{1} \wedge i \in \mathbb{N} \wedge F_{1}\left(t_{1}, s\right)>i\right\}$. Furthermore, extend $\rho_{1}$ to $\rho_{1}^{\prime}$ by $\rho_{1}^{\prime}(\mathfrak{t}):=t_{1}$ and $\rho_{1}^{\prime}\left(s_{i}\right):=s$. Then $\rho_{1}^{\prime}(\mathfrak{t})={ }^{\bullet} t_{1}=\rho_{1}^{\prime}(\bullet \mathfrak{t})$ and $\rho_{1}^{\prime}(\mathfrak{t})^{\bullet}=t_{1} \bullet=\rho_{1}^{\prime}\left(\mathfrak{t}^{\bullet}\right)$, so $\left(\mathcal{N}^{\prime}, \rho_{1}^{\prime}\right)$ is a process of $N_{1}$, extending $\left(\mathcal{N}, \rho_{1}\right)$. Since $\mathscr{R}$ is a process-based spbisimulation, $N_{2}$ has a process $\left(\mathcal{N}^{\prime}, \rho_{2}^{\prime}\right) \geq\left(\mathcal{N}, \rho_{2}\right)$ such that $\left(\rho_{1}^{\prime}, \mathcal{N}^{\prime}, \rho_{2}^{\prime}\right) \in \mathscr{R}$.

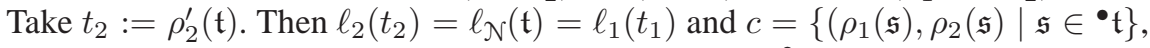

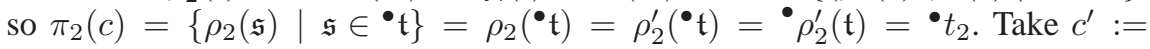
$\left\{\left(\rho_{1}^{\prime}(\mathfrak{s}), \rho_{2}^{\prime}(\mathfrak{s})\right) \mid \mathfrak{s} \in \mathfrak{t}^{\bullet}\right\}$. Then $\pi_{1}\left(c^{\prime}\right)=t_{1}^{\bullet}, \pi_{2}\left(c^{\prime}\right)=t_{2} \bullet$ and $l^{\prime}:=l-c+c^{\prime}=$

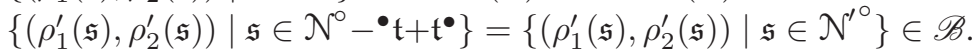

- The other clause follows by symmetry.

Since $\mathscr{R}$ contains a triple $\left(\rho_{1}, \mathcal{N}, \rho_{2}\right)$ with $\mathcal{N}$ a causal net containing no transitions, $\mathscr{B}$ contains a linking $l:=\left\{\left(\rho_{1}(\mathfrak{s}), \rho_{2}(\mathfrak{s})\right) \mid \mathfrak{s} \in \mathcal{N}^{\circ}\right.$ such that $\pi_{i}(l)=\rho_{i}\left(\mathcal{N}^{\circ}\right)=M_{i}$ for $i=1,2$, where $M_{i}$ is the initial marking of $N_{i}$. Since $\left(\mathcal{N}, \rho_{i}\right)$ is a process of $N_{i}, N_{i}$

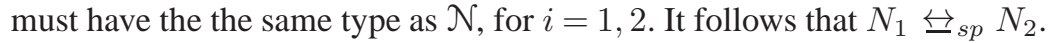

Now let $\mathscr{B}$ be an sp-bisimulation between nets $N_{1}$ and $N_{2}$. Let $\mathscr{R}:=\left\{\left(\rho_{1}, \mathcal{N}, \rho_{2}\right) \mid\right.$ $\left(\mathcal{N}, \rho_{i}\right)$ is a finite process of $N_{i}(i=1,2)$ and $\left.\left\{\left(\rho_{1}(\mathfrak{s}), \rho_{2}(\mathfrak{s})\right) \mid \mathfrak{s} \in \mathcal{N}^{\circ}\right\} \in \mathscr{B}\right\}$. Then $\mathscr{R}$ is a process-based sp-bisimulation.

- $\mathscr{B}$ must contain a linking $l$ with $\pi_{i}(l)=M_{i}$ for $i=1,2$, where $M_{i}$ is the initial marking of $N_{i}$; let $l=\left\{\left(s_{1}^{k}, s_{2}^{k}\right) \mid k \in K\right\}$. Let $\mathcal{N}$ be a causal net with places $\mathfrak{s}^{k}$ for $k \in K$ and no transitions, and define $\rho_{i}$ for $i=1,2$ by $\rho_{i}\left(\mathfrak{s}^{k}\right)=s_{i}^{k}$ for $k \in K$. Then $\left(\mathcal{N}, \rho_{i}\right)$ is an initial process of $N_{i}(i=1,2)$ and $\left(\rho_{1}, \mathcal{N}, \rho_{2}\right) \in \mathscr{R}$.

- Suppose $\left(\rho_{1}, \mathcal{N}, \rho_{2}\right) \in \mathscr{R}$ and $\left(\mathcal{N}^{\prime}, \rho_{1}^{\prime}\right)$ is a finite process of $N_{1}$ extending $\left(\mathcal{N}, \rho_{1}\right)$. (The case of a finite process of $N_{2}$ extending $\left(\mathcal{N}, \rho_{1}\right)$ will follow by symmetry.) Then $l:=\left\{\left(\rho_{1}(\mathfrak{s}), \rho_{2}(\mathfrak{s})\right) \mid \mathfrak{s} \in \mathcal{N}^{\circ}\right\} \in \mathscr{B}$. Without loss of generality, I may assume that $\mathcal{N}^{\prime}$ extends $\mathcal{N}$ by just one transition, $t$. The definition of a causal net ensures that $\bullet \mathfrak{t} \subseteq \mathcal{N}^{\circ}$, and the definition of a process gives $\rho_{1}^{\prime}(\bullet \mathfrak{t})={ }^{\bullet} t_{1}$, where $t_{1}:=\rho_{1}^{\prime}(\mathfrak{t})$. Let

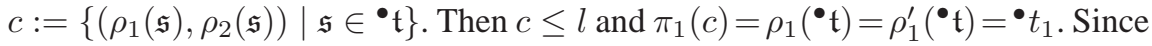
$\mathscr{B}$ is an sp-bisimulation, there are a transition $t_{2}$ with $\ell\left(t_{2}\right)=\ell\left(t_{1}\right)$ and $\pi_{2}(c)=\bullet t_{2}$, and a linking $c^{\prime}$ such that $\pi_{1}\left(c^{\prime}\right)=t_{1} \bullet, \pi_{2}\left(c^{\prime}\right)=t_{2}{ }^{\bullet}$ and $l^{\prime}:=l-c+c^{\prime} \in \mathscr{B}$. The definition of a process gives $\rho_{1}^{\prime}\left(\mathfrak{t}^{\bullet}\right)=t_{1}$. This makes it possible to extend $\rho_{2}$ to $\rho_{2}^{\prime}$ so that $\rho_{2}^{\prime}(\mathfrak{t}):=t_{2}, \rho_{2}^{\prime}\left(\mathfrak{t}^{\bullet}\right)=t_{2} \bullet$ and $c^{\prime}=\left\{\left(\rho_{1}^{\prime}(\mathfrak{s}), \rho_{2}^{\prime}(\mathfrak{s})\right) \mid \mathfrak{s} \in \mathfrak{t}^{\bullet}\right\}$. Moreover, $\rho_{2}^{\prime}(\bullet \mathfrak{t})=\rho_{2}(\bullet \mathfrak{t})=\pi_{2}(c)=\bullet t_{2}$. Thus $\left(\mathcal{N}^{\prime}, \rho_{2}^{\prime}\right)$ is a finite process of $N_{2}$ extending $\left(\mathcal{N}, \rho_{2}\right)$. Furthermore, $\left\{\left(\rho_{1}^{\prime}(\mathfrak{s}), \rho_{2}^{\prime}(\mathfrak{s})\right) \mid \mathfrak{s} \in \mathcal{N}^{\prime \circ}\right\}=\left\{\left(\rho_{1}^{\prime}(\mathfrak{s}), \rho_{2}^{\prime}(\mathfrak{s})\right) \mid\right.$ $\left.\mathfrak{s} \in \mathcal{N}^{\circ}-\bullet \mathfrak{t}+\mathfrak{t}^{\bullet}\right\}=l-c+c^{\prime} \in \mathscr{B}$. Hence $\left(\rho_{1}^{\prime}, \mathcal{N}^{\prime}, \rho_{2}^{\prime}\right) \in \mathscr{R}$. 


\section{Relating sp-bisimilarity to other semantic equivalences}

In this section I place sp-bisimilarity in the spectrum of existing semantic equivalences for nets, as indicated in Figure1

\subsection{Place bisimilarity}

The notion of a place bisimulation, defined in [1], can be reformulated as follows.

Definition 10. A place bisimulation is a structure preserving bisimulation of the form $\overline{\mathcal{B}}$ (where $\overline{\mathcal{B}}$ is defined in Section 5). Two nets $N_{i}=\left(S_{i}, T_{i}, F_{i}, M_{i}, A_{i}, \ell_{i}\right)(i=1,2)$ are strongly bisimilar, notation $N_{1} \approx_{p b} N_{2}$, if $A_{1}=A_{2}$ and there is a linking $l$ in a place bisimulation with $M_{1}=\pi_{1}(l)$ and $M_{2}=\pi_{2}(l)$.

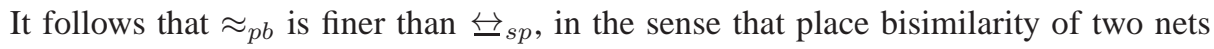
implies their structure preserving bisimilarity.

\subsection{Occurrence net equivalence}

Definitions of the unfolding for various classes of Petri nets into an occurrence net appear in $[25[35] 36|16| 7|23| 12]$ - I will not repeat them here. In all these cases, the definition directly implies that if an occurrence net $\mathcal{N}$ results from unfolding a net $N$ then $\mathcal{N}$ is safe and there exists a folding of $\mathcal{N}$ into $N$ (recall Definition 6 satisfying

- if $\mathcal{M}$ is a reachable marking of $\mathcal{N}$, and $t \in T$ is a transition of $N$ with ${ }^{\bullet} t \leq \rho(\mathcal{M})$ then there is a $\mathfrak{t} \in \mathcal{T}$ with $\rho(\mathfrak{t})=t$.

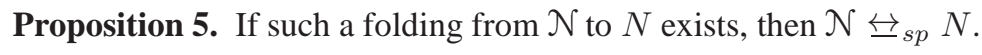

Proof. The set of linkings $\mathscr{B}:=\{\{(\mathfrak{s}, \rho(\mathfrak{s})) \mid \mathfrak{s} \in \mathcal{M}\} \mid \mathcal{M}$ a reachable marking of $\mathcal{N}\}$ is an sp-bisimulation between $\mathcal{N}$ and $N$. Checking this is trivial.

Two nets $N_{1}$ and $N_{2}$ are occurrence net equivalent [16] if they have isomorphic unfoldings. Since isomorphic nets are strongly bisimilar [27] and hence structure preserving bisimilar, it follows that occurrence net equivalence between nets is finer than structure preserving bisimilarity.

In [1] it is pointed out that the strong bisimilarity of Olderog "is not compatible with unfoldings": they show two nets that have isomorphic unfoldings, yet are not strongly bisimilar. However, when the net $N$ is safe, the sp-bisimulation displayed in the proof of Proposition 5 is in fact a strong bisimulation, showing that each net is strongly bisimilar with its unfolding. This is compatible with the observation of [1] because of the nontransitivity of strong bisimilarity. 


\subsection{Causal equivalence}

Causal equivalence is coarser than structure preserving bisimilarity.

Theorem 4. If $N_{1} \overleftrightarrow{\unlhd}_{s p} N_{2}$ for nets $N_{1}$ and $N_{2}$, then $N_{1} \equiv_{\text {caus }} N_{2}$.

Proof. By Theorem 3 there exists a process-based sp-bisimulation $\mathscr{R}$ between $N_{1}$ and $N_{2}$. $\mathscr{R}$ must contain a triple $\left(\rho_{1}^{0}, \mathcal{N}^{0}, \rho_{2}^{0}\right)$ with $\mathcal{N}^{0}$ a causal net containing no transitions. So $\left(\mathcal{N}^{0}, \rho_{1}^{0}\right)$ and $\left(\mathcal{N}^{0}, \rho_{2}^{0}\right)$ are initial processes of $N_{1}$ and $N_{2}$, respectively. The net $\mathcal{N}^{0}$ contains isolated places only, as many as the size of the initial markings of $N_{1}$ and $N_{2}$.

Let $\mathcal{N}$ be a causal net of $N_{1}$. I have to prove that $\mathcal{N}$ is also a causal net of $N_{2}$. Without loss of generality I may assume that $\mathcal{N}^{0}$ is a prefix of $\mathcal{N}$, as being a causal net of a given Petri net is invariant under renaming of its places and transitions.

So $N_{1}$ has a process $\mathcal{P}_{1}=\left(\mathcal{N}, \rho_{1}\right)$. By Proposition $4 \mathcal{P}_{1}$ is the limit of a chain $\mathcal{P}_{1}^{0} \leq \mathcal{P}_{1}^{1} \leq \mathcal{P}_{1}^{2} \leq \ldots$ of finite processes of $N_{1}$. Moreover, for $\mathcal{P}_{1}^{0}$ one can take $\left(\mathcal{N}^{0}, \rho_{1}^{0}\right)$. Let $\mathcal{P}_{1}^{i}=\left(\mathcal{N}^{i}, \rho_{1}^{i}\right)$ for $i \in \mathbb{N}$. By induction on $i \in \mathbb{N}$, it now follows from the properties of a process-based sp-bisimulation that $N_{2}$ has processes $\mathcal{P}_{2}^{i+1}=\left(\mathcal{N}^{i+1}, \rho_{2}^{i+1}\right)$, such that $\left(\mathcal{N}^{i}, \rho_{2}^{i}\right) \leq\left(\mathcal{N}^{i+1}, \rho_{2}^{i+1}\right)$ and $\left(\rho_{1}^{i+1}, \mathcal{N}^{i+1}, \rho_{2}^{i+1}\right) \in \mathscr{R}$. Using Proposition 3 , the limit $\mathcal{P}_{2}=\left(\mathcal{N}, \rho_{2}\right)$ of this chain is a process of $N_{2}$, contributing the causal net $\mathcal{N}$.

\subsection{History preserving bisimilarity}

The notion of history preserving bisimilarity was originally proposed in [32] under the name behavior structure bisimilarity, studied on event structures in [13], and first defined on Petri nets, under to the individual token interpretation, in [2], under the name fully concurrent bisimulation equivalence.

Definition 11. [2] Let $\mathcal{N}_{i}=\left(\mathcal{S}_{i}, \mathcal{T}_{i}, \mathcal{F}_{i}, \mathcal{M}_{0 i}, \mathcal{A}_{i}, \ell_{i}\right)(i=1,2)$ be two causal nets. An order-isomorphism between them is a bijection $\beta: \mathcal{T}_{1} \rightarrow \mathcal{T}_{2}$ such that $\mathcal{A}_{1}=\mathcal{A}_{2}$, $\ell_{2}(\beta(t))=\ell_{1}(t)$ for all $t \in \mathcal{T}_{1}$, and $t \mathcal{F}_{1}^{+} u$ iff $\beta(t) \mathcal{F}_{2}^{+} \beta(u)$ for all $t, u \in T_{1}$.

Definition 12. [2] A fully concurrent bisimulation between two nets $N_{1}$ and $N_{2}$ is a set $\mathscr{R}$ of triples $\left(\left(\rho_{1}, \mathcal{N}_{1}\right), \beta,\left(\mathcal{N}_{2}, \rho_{2}\right)\right)$ with $\left(\mathcal{N}_{i}, \rho_{i}\right)$ a finite process of $N_{i}$, for $i=1,2$, and $\beta$ an order-isomorphism between $\mathcal{N}_{1}$ and $\mathcal{N}_{2}$, such that

- $\mathscr{R}$ contains a triple $\left(\left(\rho_{1}, \mathcal{N}_{1}\right), \beta,\left(\mathcal{N}_{2}, \rho_{2}\right)\right)$ with $\mathcal{N}_{1}$ containing no transitions,

- if $\left(\mathcal{P}_{1}, \beta, \mathcal{P}_{2}\right) \in \mathscr{R}$ and $\mathcal{P}_{i}^{\prime}$ with $i \in\{1,2\}$ is a fin. proc. of $N_{i}$ extending $\mathcal{P}_{i}$, then $N_{j}$ with $j:=3-i$ has a process $\mathcal{P}_{j}^{\prime} \geq \mathcal{P}_{j}$ such that $\left(\mathcal{P}_{1}^{\prime}, \beta^{\prime}, \mathcal{P}_{2}^{\prime}\right) \in \mathscr{R}$ for some $\beta^{\prime} \supseteq \beta$.

Write $N_{1} \approx_{h} N_{2}$ or $N_{1} \approx_{f c b} N_{2}$ iff such a bisimulation exists.

It follows immediately from the process-based characterisation of sp-bisimilarity in Section 9 that fully concurrent bisimilarity (or history preserving bisimilarity based on the individual token interpretation of nets) is coarser than sp-bisimilarity.

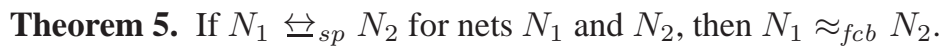

Proof. A process-based sp-bisimulation is simply a fully concurrent bisimulation with the extra requirement that $\beta$ must be the identity relation. 


\section{Inevitability for non-reactive systems}

A run or execution of a system modelled as Petri net $N$ can be formalised as a path of $N$ (defined in Section 3 ) or a process of $N$ (defined in Section 8). A path or process representing a complete run of the represented system-one that is not just the first part of a larger run-is sometimes called a complete path or process. Once a formal definition of a complete path or process is agreed upon, an action $b$ is inevitable in a net $N$ iff each complete path (or each complete process) of $N$ contains a transition labelled $b$. In case completeness is defined both for paths and processes, the definitions ought to be such that they give rise to the same concept of inevitability.

The definition of which paths or processes count as being complete depends on two factors: (1) whether actions that a net can perform by firing a transition are fully under control of the represented system itself or (also) of the environment in which it will be running, and (2) what type of progress or fairness assumption one postulates to guarantee that actions that are due to occur will actually happen. In order to address (2) first, in this section I deal only with nets in which all activity is fully under control of the represented system. In Section 14 I will generalise the conclusions to reactive systems.

When making no progress or fairness assumptions, a system always has the option not to progress further, and all paths and all processes are complete-in particular initial paths and processes, containing no transitions. Consequently, no action is inevitable in any net, so each semantic equivalence respects inevitability.

When assuming progress, but not justness or fairness, any infinite path or process is complete, and a finite path or process is complete iff it is maximal, in the sense that it has no proper extension. In this setting, interleaving bisimilarity, and hence also each finer equivalence, respects inevitability. The argument is that an interleaving bisimulation induces a relation between the paths of two related nets $N_{1}$ and $N_{2}$, such that

- each path of $N_{1}$ is related to a path of $N_{2}$ and vice versa,

- if two paths are related, either both or neither contain a transition labelled $b$,

- if two paths are related, either both or neither of them are complete.

In the rest of this paper I will assume justness, and hence also progress, but not (weak or strong) fairness, as explained in Section 1.4 In this setting a process is just or complet 4 iff it is maximal, in the sense that it has no proper extension.

Example 3. The net depicted on the right has a complete process performing the action $a$ infinitely often, but never the action $b$. It consumes each to-

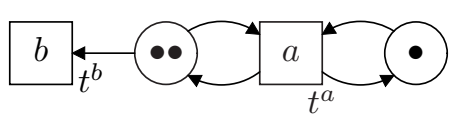
ken that is initially present or stems from any firing of the transition $t^{a}$. Hence $b$ is not inevitable. This fits with the intuition that if a transition occurrence is perpetually enabled it will eventually happen-but only when strictly adhering to the individual token interpretation of nets. Under this interpretation, each firing of $t^{b}$ using a particular token is a different transition occurrence. It is possible to schedule an infinite sequence of $a \mathrm{~s}$ in such a way that none such transition occurrence is perpetually enabled from some point onwards.

\footnotetext{
${ }^{4}$ The term "complete" is meant to vary with the choice of a progress or fairness assumption; when assuming only justness, it is set to the value "just".
} 
When adhering to the collective token interpretation of nets, the action $b$ could be considered inevitable, as in any execution scheduling $a$ s only, transition $t^{b}$ is perpetually enabled. Since my structure preserving bisimulation fits within the individual token interpretation, here one either should adhere to that interpretation, or restrict attention to safe nets, where there is no difference between both interpretations.

\section{History preserving bisimilarity does not respect inevitability}

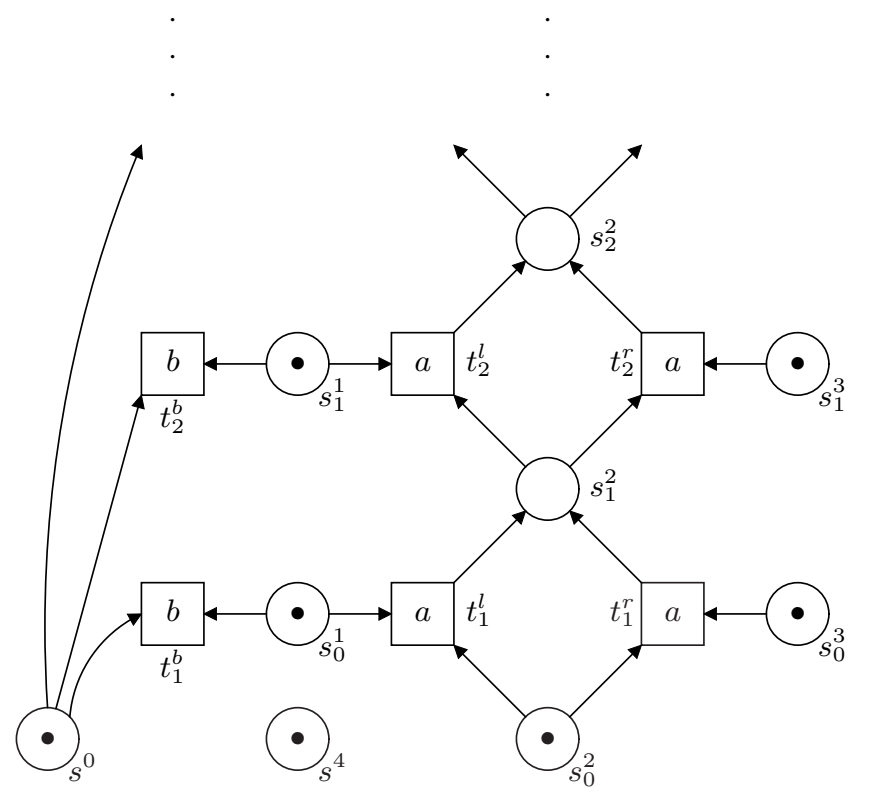

Fig. 5. A net in which the action $b$ is not inevitable

Consider the safe net $N_{1}$ depicted in Figure 5 , and the net $N_{2}$ obtained from $N_{1}$ by exchanging for any transition $t_{i}^{b}(i>0)$ the preplace $s_{i-1}^{1}$ for $s^{4}$. The net $N_{2}$ performs in parallel an infinite sequence of $a$-transitions (where at each step $i>0$ there is a choice between $t_{i}^{l}$ and $t_{i}^{r}$ ) and a single $b$-transition (where there is a choice between $t_{i}^{b}$ for $i>0$ ). In $N_{2}$ the action $b$ is inevitable. In $N_{1}$, on the other hand, $b$ is not inevitable, for the run of $N_{1}$ in which $t_{i}^{l}$ is chosen over $t_{i}^{r}$ for all $i>0$ is complete, and cannot be extended which a $b$-transition. Thus, each semantic equivalence that equates $N_{1}$ and $N_{2}$ fails to respect inevitability.

Theorem 6. Causal equivalence does not respect inevitability.

Proof. $N_{1} \equiv_{\text {caus }} N_{2}$, because both nets have the same causal nets. One of these nets is depicted in Figure 6, the others are obtained by omitting the $b$-transition, and/or omitting all but a finite prefix of the $a$-transitions. 

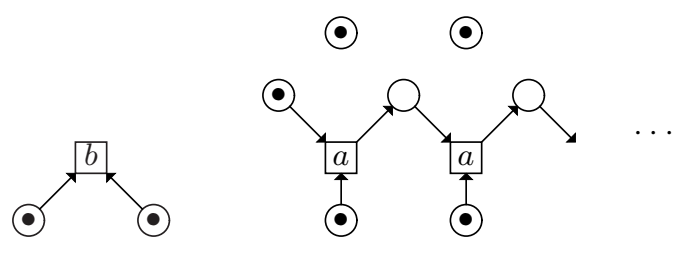

Fig. 6. A causal net of $N_{1}$ and $N_{2}$

Theorem 7. History preserving bisimilarity does not respect inevitability.

Proof. Recall that $N_{1}$ and $N_{2}$ differ only in their flow relations, and have the same set of transitions. I need to describe a fully concurrent bisimulation $\mathscr{R}$ between $N_{1}$ and $N_{2}$. $\mathscr{R}$ consists of a set of triples, each consisting of a process of $N_{1}$, a related process of $N_{2}$, and an order isomorphism between them. First of all I include all triples $\left(\mathcal{P}_{1}, \beta, \mathcal{P}_{2}\right)$ where $\mathcal{P}_{1}$ is an arbitrary process of $N_{1}, \mathcal{P}_{2}$ is the unique process of $N_{2}$ that induces the same set of transitions as $\mathcal{P}_{1}$, and $\beta$ relates transition of $\mathcal{P}_{1}$ and $\mathcal{P}_{2}$ when they map to the same transition of $N_{i}(i=1,2)$. Secondly, I include all triples $\left(\mathcal{P}_{1}, \beta, \mathcal{P}_{2}\right)$ where $\mathcal{P}_{2}$ is an arbitrary process of $N_{2}$ inducing both $t_{k}^{b}$ and $t_{k}^{l}$ for some $k>0$, and $\mathcal{P}_{1}$ is any process of $N_{1}$ that induces the same transitions as $\mathcal{P}_{2}$ except that, for some $h \geq k$ the induced transition $t_{h}^{l}$, if present, is replaced by $t_{h}^{r}$, and $t_{k}^{b}$ is replaced by $t_{h}^{b}$. ( $\beta$ should be obvious.) It is trivial to check that the resulting relation is a fully concurrent bisimulation indeed.

\section{Structure preserving bisimilarity respects inevitability}

Definition 13. A net $\mathcal{N}$ is called a complete causal net of a net $N$ if it is the first component of a maximal process $(\mathcal{N}, \rho)$ of $N$. Two nets $N_{1}$ and $N_{2}$ are complete causal net equivalent, notation $\equiv_{c c}$, if they have the same complete causal nets.

Since the causal nets of a net $N$ are completely determined by the complete causal nets of $N$, namely as their prefixes, $N_{1} \equiv_{c c} N_{2}$ implies $N_{1} \equiv_{\text {caus. }} N_{2}$. It follows immediately from the definition of inevitability that $\equiv_{c c}$ respects inevitability. Thus, to

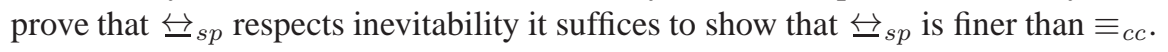

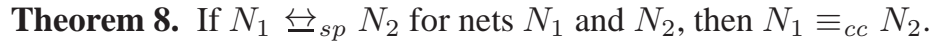

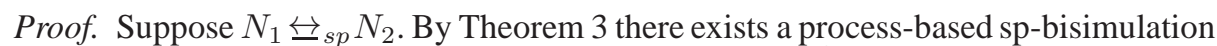
$\mathscr{R}$ between $N_{1}$ and $N_{2} . \mathscr{R}$ must contain a triple $\left(\rho_{1}^{0}, \mathcal{N}^{0}, \rho_{2}^{0}\right)$ with $\mathcal{N}^{0}$ a causal net containing no transitions. So $\left(\mathcal{N}^{0}, \rho_{1}^{0}\right)$ and $\left(\mathcal{N}^{0}, \rho_{2}^{0}\right)$ are initial processes of $N_{1}$ and $N_{2}$, respectively. The net $\mathcal{N}^{0}$ contains isolated places only.

Let $\mathcal{N}$ be a complete causal net of $N_{1}$. I have to prove that $\mathcal{N}$ is also a complete causal net of $N_{2}$. Without loss of generality I may assume that $\mathcal{N}^{0}$ is a prefix of $\mathcal{N}$, as being a complete causal net of a given Petri net is invariant under renaming of its places.

So $N_{1}$ has a complete process $\mathcal{P}_{1}=\left(\mathcal{N}, \rho_{1}\right)$. By Proposition $4, \mathcal{P}_{1}$ is the limit of a chain $\mathcal{P}_{1}^{0} \leq \mathcal{P}_{1}^{1} \leq \mathcal{P}_{1}^{2} \leq \ldots$ of finite processes of $N_{1}$. Moreover, for $\mathcal{P}_{1}^{0}$ one 
can take $\left(\mathcal{N}^{0}, \rho_{1}^{0}\right)$. Let $\mathcal{P}_{1}^{i}=\left(\mathcal{N}^{i}, \rho_{1}^{i}\right)$ for $i \in \mathbb{N}$. By induction on $i \in \mathbb{N}$, it now follows from the properties of a process-based sp-bisimulation that $N_{2}$ has processes $\mathcal{P}_{2}^{i+1}=\left(\mathcal{N}^{i+1}, \rho_{2}^{i+1}\right)$, such that $\left(\mathcal{N}^{i}, \rho_{2}^{i}\right) \leq\left(\mathcal{N}^{i+1}, \rho_{2}^{i+1}\right)$ and $\left(\rho_{1}^{i+1}, \mathcal{N}^{i+1}, \rho_{2}^{i+1}\right) \in \mathscr{R}$. Using Proposition 3, the limit $\mathcal{P}_{2}=\left(\mathcal{N}, \rho_{2}\right)$ of this chain is a process of $N_{2}$. It remains to show that $\mathcal{P}_{2}$ is complete.

Towards a contradiction, let $\mathcal{P}_{2 u}=\left(\mathcal{N}_{u}, \rho_{2 u}\right)$ be a proper extension of $\mathcal{P}_{2}$, say with just one transition, $u$. Then $\bullet \subseteq \mathcal{N}^{\circ}$. By the third requirement on occurrence nets of Definition 6 their are only finitely many transitions $t$ with $(t, u) \in \mathcal{F}_{2 u}^{+}$. Hence one of the finite approximations $\mathcal{N}^{k}$ of $\mathcal{N}$ contains all these transitions. So $u \subseteq\left(\mathcal{N}^{k}\right)^{\circ}$. Let, for all $i \geq k, \mathcal{P}_{2 u}^{i}=\left(\mathcal{N}_{u}^{i}, \rho_{2 u}^{i}\right)$ be the finite prefix of $\mathcal{P}_{2 u}$ that extends $\mathcal{P}_{2}^{i}$ with the single transition $u$. Then $\mathcal{P}_{2 u}^{i} \leq \mathcal{P}_{2 u}^{i+1}$ for all $i \geq k$, and the limit of the chain $\mathcal{P}_{2 u}^{k} \leq \mathcal{P}_{2 u}^{k+1} \leq \ldots$ is $\mathcal{P}_{2 u}$. By induction on $i \in \mathbb{N}$, it now follows from the properties of a process-based sp-bisimulation that $N_{1}$ has processes $\mathcal{P}_{1 u}^{i}=\left(\mathcal{N}_{u}^{i}, \rho_{1 u}^{i}\right)$ for all $i \geq k$, such that $\left(\rho_{1 u}^{i}, \mathcal{N}_{u}^{i}, \rho_{2 u}^{i}\right) \in \mathscr{R},\left(\mathcal{N}^{k}, \rho_{1}^{k}\right) \leq\left(\mathcal{N}_{u}^{k}, \rho_{1 u}^{k}\right)$ and $\left(\mathcal{N}_{u}^{i}, \rho_{1 u}^{i}\right) \leq\left(\mathcal{N}_{u}^{i+1}, \rho_{1 u}^{i+1}\right)$. Using Proposition 3] the limit $\mathcal{P}_{1 u}=\left(\mathcal{N}_{u}, \rho_{1 u}\right)$ of this chain is a process of $N_{1}$. It extends $\mathcal{P}_{1}$ with the single transition $u$, contradicting the maximality of $\mathcal{P}_{1}$.

\section{Inevitability for reactive systems}

In the modelling of reactive systems, an action performed by a net is typically a synchronisation between the net itself and its environment. Such an action can take place only when the net is ready to perform it, as well as its environment. In this setting, an adequate formalisation of the concepts of justness and inevitability requires keeping track of the set of actions that from some point onwards are blocked by the environment-e.g. because the environment is not ready to partake in the synchronisation. Such actions are not required to occur eventually, even when they are perpetually enabled by the net itself. Let's speak of a $Y$-environment if $Y$ is this set of actions. In Section 11]I restricted attention to $\emptyset$-environments, in which an action can happen as soon as it is enabled by the net in question. In [15] a path is called $Y$-just iff, when assuming justness, it models a complete run of the represented system in a $Y$-environment. The below is a formalisation for this concept for Petri nets under the individual token interpretation.

Definition 14. A process of a net is $Y$-just or $Y$-complete it each of its proper extensions adds a transition with a label in $Y$.

Note that a just or complete process as defined in Section 11 is a $\emptyset$-just or $\emptyset$-complete process. In applications there often is a subset of actions that are known to be fully controlled by the system under consideration, and not by its environment. Such actions are often called non-blocking. A typical example from process algebra [24] is the internal action $\tau$. In such a setting, $Y$-environments exists only for sets of actions $Y \subseteq \mathscr{C}$, where $\mathscr{C}$ is the set of all non-non-blocking actions.

A process of a net is complete if it models a complete run of the represented system in some environment. This is the case iff it is $Y$-complete for some set $Y \subseteq \mathscr{C}$, which is the case iff it is $\mathscr{C}$-complete.

In [34], non-blocking is a property of transitions rather than actions, and nonblocking transitions are called hot. Transitions that are not hot are cold, which inspired 
my choice of the latter $\mathscr{C}$ above. In this setting, a process $\mathcal{P}=(\mathcal{N}, \rho)$ is complete iff the marking $\rho\left(\mathcal{N}^{\circ}\right)$ enables cold transitions only [34].

Definition 15. A action $b$ is $Y$-inevitable in a net if each $Y$-complete process contains a transition labelled $b$. A semantic equivalence $\approx$ respects $Y$-inevitability if whenever $N_{1} \approx N_{2}$ and $b$ is $Y$-inevitable in $N_{1}$, then $b$ is $Y$-inevitable in $N_{2}$. It respects inevitability iff it respects $Y$-inevitability for each $Y \subseteq \mathscr{C}$.

In Section 12 it is shown that $\equiv_{\text {caus }}$ and $\approx_{h}$ do not respect $\emptyset$-inevitability. From this

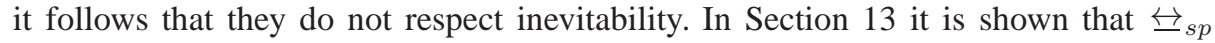
does respect $\emptyset$-inevitability. By means of a trivial adaptation the same proof shows that $\overleftrightarrow{ }_{s p}$ respects $Y$-inevitability, for arbitrary $Y$. All that is needed is to assume that the

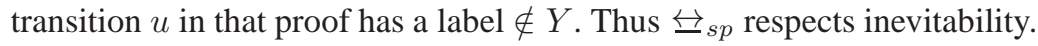

\section{Conclusion}

This paper proposes a novel semantic equivalence for current systems represented as Petri nets: structure preserving bisimilarity. As a major application-the one that inspired this work-it is used to establish the agreement between the operational Petri net semantics of the process algebra CCSP as proposed by Olderog, and its denotational counterpart. An earlier semantic relation used for this purpose was Olderog's strong bisimilarity on safe Petri nets, but that relation failed to be transitive. I hereby conjecture that on the subclass of occurrence nets, strong bisimilarity and structure preserving bisimilarity coincide. If this it true, it follows, together with the observations of Section 6 that strong bisimilarity is included in structure preserving bisimilarity, and of Section 10.2 that each safe net is strongly bisimilar with its unfolding into an occurrence net, that on safe nets structure preserving bisimilarity is the transitive closure of strong bisimilarity.

Section 1.2 proposes nine requirements on a semantic equivalence that is used for purposes like the one above. I have shown that structure preserving bisimilarity meets eight of these requirements and conjecture that it meets the remaining one as well.

- It meets Requirement 1, that it respects branching time, as a consequence of Theorem 5, saying that it is finer than history preserving bisimilarity, which is known to be finer than interleaving bisimilarity.

- It meets Requirement 2, that it fully captures causality and concurrency (and their interplay with branching time) 5 also as a consequence of Theorem 5

- It meets Requirement 3, that it respects inevitability (under the standard interpretation of Petri nets that assumes justness but not fairness), ${ }^{5}$ as shown in Section 13

- It meets Requirement 4, that it is real-time consistent, as a result of Theorem5

- I conjecture that it meets Requirement 5, that it is preserved under action refinement.

- It meets Requirement 6, that it is finer than causal equivalence, by Theorem 4 .

- It meets Requirement 7, that it is coarser than $\equiv_{o c c}$, as shown in Section 10.2 .

- It meets Requirement 8, that it is a congruence for the CCSP operators, by Thm.2

- It meets Requirement 9, that it allows to establish agreement between the operational and denotational interpretations of CCSP operators, since it is coarser than Olderog's strong bisimilarity, as shown in Section 6

\footnotetext{
${ }^{5}$ when taking the individual token interpretation of nets, or restricting attention to safe ones
} 
Moreover, structure preserving bisimilarity is the first known equivalence that meets these requirements. In fact, it is the first that meets the key Requirements 3, 4, 7 and 9.

Acknowledgement My thanks to Ursula Goltz for proofreading and valuable feedback.

\section{References}

1. C. Autant, Z. Belmesk \& P. Schnoebelen (1991): Strong Bisimilarity on Nets Revisited. In E.H.L. Aarts, J. van Leeuwen \& M. Rem, editors: Proc. PARLE '91, Eindhoven, The Netherlands, 1991, LNCS 506, Springer, pp. 295-312, doi:10.1007/3-540-54152-7_71.

2. E. Best, R. Devillers, A. Kiehn \& L. Pomello (1991): Concurrent Bisimulations in Petri nets. Acta Informatica 28, pp. 231-264, doi:10.1007/BF 01178506.

3. S.D. Brookes, C.A.R. Hoare \& A.W. Roscoe (1984): A theory of communicating sequential processes. Journal of the ACM 31(3), pp. 560-599, doi:10.1145/828.833.

4. L. Castellano, G. De Michelis \& L. Pomello (1987): Concurrency vs interleaving: an instructive example. Bulletin of the EATCS 31, pp. 12-15.

5. P. Degano, R. De Nicola \& U. Montanari (1987): CCS is an (Augmented) Contact Free C/E System. In M.V. Zilli, editor: Mathematical Models for the Semantics of Parallelism, LNCS 280, Springer, pp. 144-165, doi:10.1007/3-540-18419-8_13.

6. E.A. Emerson \& E.M. Clarke (1982): Using Branching Time Temporal Logic to Synthesize Synchronization Skeletons. Science of Computer Programming 2(3), pp. 241-266, doi:10. 1016/0167-6423(83) 90017-5.

7. J. Engelfriet (1991): Branching Processes of Petri Nets. Acta Informatica 28(6), pp. 575591, doi:10.1007/BF01463946.

8. A. Fehnker, R.J. van Glabbeek, P. Höfner, A.K. McIver, M. Portmann \& W.L. Tan (2013): A Process Algebra for Wireless Mesh Networks used for Modelling, Verifying and Analysing AODV. Technical Report 5513, NICTA, Sydney, Australia. Available at http: / / arxiv . org/abs/1312.7645.

9. H. Genrich \& E. Stankiewicz-Wiechno (1980): A Dictionary of Some Basic Notions of Net Theory. In W. Brauer, editor: Advanced Course: Net Theory and Applications, LNCS 84, Springer, pp. 519-531, doi:10.1007/3-540-10001-6_39.

10. R.J. van Glabbeek (1990): The refinement theorem for ST-bisimulation semantics. In M. Broy \& C.B. Jones, editors: Proceedings IFIP TC2 Working Conference on Programming Concepts and Methods, Sea of Gallilee, Israel, April 1990, North-Holland, pp. $27-52$.

11. R.J. van Glabbeek (2001): The Linear Time - Branching Time Spectrum I; The Semantics of Concrete, Sequential Processes. In J.A. Bergstra, A. Ponse \& S.A. Smolka, editors: Handbook of Process Algebra, chapter 1, Elsevier, pp. 3-99, doi:10.1016/ B978-044482830-9/50019-9.

12. R.J. van Glabbeek (2005): The Individual and Collective Token Interpretations of Petri Nets. In M. Abadi \& L. de Alfaro, editors: Proc. CONCUR 2005, San Francisco, USA, August 2005, LNCS 3653, Springer, pp. 323-337, doi:10.1007/11539452_26.

13. R.J. van Glabbeek \& U. Goltz (2001): Refinement of Actions and Equivalence Notions for Concurrent Systems. Acta Informatica 37, pp. 229-327, doi:10.1007/s 002360000041.

14. R.J. van Glabbeek \& P. Höfner (2015): CCS: It's not fair! Acta Informatica 52(2-3), pp. 175-205, doi:10.1007/s00236-015-0221-6.

15. R.J. van Glabbeek \& P. Höfner (2015): Progress, Fairness and Justness in Process Algebra. Available at http://arxiv.org/abs/1501.03268.

16. R.J. van Glabbeek \& F.W. Vaandrager (1987): Petri net models for algebraic theories of concurrency (extended abstract). In J.W. de Bakker, A.J. Nijman \& P.C. Treleaven, editors: Proc. PARLE, LNCS 259, Springer, pp. 224-242, doi:10.1007/3-540-17945-3_13. 
17. R.J. van Glabbeek \& F.W. Vaandrager (1997): The Difference Between Splitting in $n$ and $n+1$. Information and Comput. 136(2), pp. 109-142, doi:10.1006/inco.1997.2634.

18. U. Goltz \& A. Mycroft (1984): On the relationship of CCS and Petri nets. In J. Paredaens, editor: Proceedings $11^{\text {th }}$ ICALP, Antwerpen, LNCS 172, Springer, pp. 196-208, doi:10 . 1007/3-540-13345-3_18.

19. U. Goltz \& W. Reisig (1983): The Non-Sequential Behaviour of Petri Nets. Information and Control 57(2-3), pp. 125-147, doi:10.1016/S0019-9958 (83) 80040-0.

20. C.A.R. Hoare (1985): Communicating Sequential Processes. Prentice Hall,Englewood Cliffs.

21. R. Loogen \& U. Goltz (1991): Modelling nondeterministic concurrent processes with event structures. Fundamenta Informaticae 14(1), pp. 39-74.

22. A.W. Mazurkiewicz, E. Ochmanski \& W. Penczek (1989): Concurrent Systems and Inevitability. TCS 64(3), pp. 281-304, doi:10.1016/0304-3975 (89) 90052-2.

23. J. Meseguer, U. Montanari \& V. Sassone (1997): On the semantics of place/transition Petri nets. Mathematical Structures in Computer Science 7(4), pp. 359-397, doi:10.1017/ S0960129597002314.

24. R. Milner (1990): Operational and algebraic semantics of concurrent processes. In J. van Leeuwen, editor: Handbook of Theoretical Computer Science, chapter 19, Elsevier Science Publishers B.V. (North-Holland), pp. 1201-1242. Alternatively see Communication and Concurrency, Prentice-Hall, Englewood Cliffs, 1989, of which an earlier version appeared as A Calculus of Communicating Systems, LNCS 92, Springer, 1980, doi:10.1007/ 3-540-10235-3.

25. M. Nielsen, G.D. Plotkin \& G. Winskel (1981): Petri nets, event structures and domains, part I. TCS 13(1), pp. 85-108, doi:10.1016/0304-3975 (81) 90112-2.

26. E.-R. Olderog (1987): Operational Petri net semantics for CCSP. In G. Rozenberg, editor: Advances in Petri Nets 1987, covers the 7th European Workshop on Applications and Theory of Petri Nets, Oxford, UK, June 1986, LNCS 266, Springer, pp. 196-223, doi:10.1007/ 3-540-18086-9_27.

27. E.-R. Olderog (1991): Nets, Terms and Formulas: Three Views of Concurrent Processes and their Relationship. Cambridge Tracts in Theor. Comp. Sc. 23, Cambridge University Press.

28. E.-R. Olderog \& C.A.R. Hoare (1986): Specification-oriented semantics for communicating processes. Acta Informatica 23, pp. 9-66, doi:10.1007/BF00268075.

29. C.A. Petri (1977): Non-sequential processes. Internal Report GMD-ISF-77.05, GMD, St. Augustin.

30. G.D. Plotkin (2004): A Structural Approach to Operational Semantics. The Journal of Logic and Algebraic Programming 60-61, pp. 17-139, doi:10.1016/j • jlap.2004.05.001. Originally appeared in 1981.

31. A. Pnueli (1977): The Temporal Logic of Programs. In: Foundations of Computer Science (FOCS '77), IEEE, pp. 46-57, doi:10 .1109/SFCS .1977.32.

32. A. Rabinovich \& B.A. Trakhtenbrot (1988): Behavior Structures and Nets. Fundamenta Informaticae 11(4), pp. 357-404.

33. W. Reisig (1985): Petri nets - an introduction. EATCS Monographs on Theoretical Computer Science, Volume 4, Springer, doi:10.1007/978-3-642-69968-9.

34. W. Reisig (2013): Understanding Petri Nets - Modeling Techniques, Analysis Methods, Case Studies. Springer, doi:10.1007/978-3-642-33278-4.

35. G. Winskel (1984): A new definition of morphism on Petri nets. In M. Fontet \& K. Mehlhorn, editors: Proceedings STACS 84, LNCS 166, Springer, pp. 140-150, doi:10. 1007/3-540-12920-0_13.

36. G. Winskel (1987): Event structures. In W. Brauer, W. Reisig \& G. Rozenberg, editors: Petri Nets: Applications and Relationships to Other Models of Concurrency, Advances in Petri Nets 1986, Part II; Proceedings of an Advanced Course, Good Honnef, September 1986, LNCS 255, Springer, pp. 325-392, doi:10.1007/3-540-17906-2_31. 